\title{
Using the Quadrupole Moment Frequency Response of Bunched Beam to Measure its Transverse Emittance
}

\author{
Cheng-Yang Tan \\ Accelerator Division/Tevatron
}

\begin{abstract}
The quadrupole moment measured with a quadrupole pickup has been used to measure the transverse emittance of the beam. Unfortunately, the poor $\mathrm{S} / \mathrm{N}$ ratio in the measurement makes it difficult to produce good and consistent emittance results. One way to enhance the $\mathrm{S} / \mathrm{N}$ is to kick the beam with a quadrupole kicker and then measure its quadrupole frequency response (QFR) with a quadrupole pickup. This paper will show that if the bunched beam is extremely short, the quadrupole tunes are well decoupled and far apart, and the quadrupole tune spread is smaller than the synchrotron tune, then the emittance can be extracted from the QFR.
\end{abstract}




\section{INTRODUCTION}

The use of a quadrupole pickup as a non-invasive method for measuring transverse

emittance is not new. ${ }^{1,2}$ However, this type of pickup has faced difficulties transitioning from an "expert only" tool to an operational tool because of the poor S/N ratio which has made it hard to get consistent and reproducible results. We quote from $\mathrm{Koziol}^{3}$ who writes about obtaining signals from quadrupole pickups: "Deriving an information that can be quantitatively interpreted is quite an art. A prerequisite is careful centering of the beam in the pick-up, otherwise the dipole oscillations will completely swamp the weak quadrupole component." It has been suggested by Cameron ${ }^{4}$ that one possible way to enhance the $\mathrm{S} / \mathrm{N}$ is to use a quadrupole kicker to excite the beam and then measure the response with a quadrupole pickup. Unfortunately, the quadrupole kicker will blow up the transverse emittance of the beam. It is intuitively obvious why this happens:

A particle which goes through the centre of the quadrupole kicker gets zero kick. However, any other particle that is off centred will experience a kick that is proportional to the distance from the centre of the quadrupole. (Compare this to a dipole kicker which has a constant size kick independent of the particle's transverse position). When this particle is kicked at its quadrupole tune the size of each kick it sees will be stronger than the last because it is moving proportionately further away from the centre. This is the recipe for producing exponential growth.

With the above in mind, we have to be careful how we define the quadrupole mode frequency response (QFR) because it does not exist if the particle moves further and further away from the centre after every turn. To overcome this problem, Guo et $a l^{5}$ calculates the emittance from the QFR for a kick that is always outside the quadrupole tune distribution (c.f. AC dipole) for coasting beam. For us, we will define the QFR for extremely weak 
kicks which only last for a very short time so that the transverse emittance blow up is minimal.

Of course, with such weak kicks, it really begs the question as to whether we are winning the $\mathrm{S} / \mathrm{N}$ battle. The 3D-BBQ electronics which will be used for measuring betatron tune at the Large Hadron Collider (LHC) and presently installed in the Super Proton Synchrotron (SPS), Relativistic Heavy Ion Collider (RHIC) and the Tevatron can see transverse motion as small as $10 \mathrm{~nm} .^{6}$ When it is installed as the electronics for a quadrupole pickup, its sensitivity should allow us to measure the QFR to the accuracy needed for a good emittance measurement.

For the rest of this paper we will set up the ground work that is necessary to find the relationship between the QFR and the emittance for bunched beam. The first requirement is for us to solve the differential equations which govern single particle motion when it is weakly kicked with a quadrupole. We then take this solution and apply it to every particle in the bunch so that the QFR can be calculated for coasting $\S$ beam. This QFR is generalised for bunched beam where we will find that for calculating emittance from the QFR, we require the quadrupole tune spread to be smaller than the synchrotron tune.

$\S$ For the purposes of this paper, we define coasting beam to be beam that has no RF structure and the relative change in momentum w.r.t. the synchronous particle $\Delta p / p=0$ so that it has no chromatic dependence. 


\section{THEORY}

We set up our system with a kicker at location $\varphi_{k}$ and the pickup at $\varphi_{p}$. See Figure 1. We note that the kicker is a quadrupole kicker, which means that it will kick in both the $x$ and the $y$ direction, i.e. it will focus the beam in one plane while simultaneously defocus it in the orthogonal plane and the quadrupole pickup will see both the $x$ and $y$ oscillations. Thus the final result must incorporate results from both transverse phase spaces $\left(x, x^{\prime}\right)$ and $\left(y, y^{\prime}\right)$. For our calculations we will first work in $\left(x, x^{\prime}\right)$ and then show how we incorporate $\left(y, y^{\prime}\right)$ into the final results.

The usual transverse phase space defined by $\left(x, x^{\prime}\right)$ is transformed into a normalised coordinates system by simply defining a new "momentum" to be

$$
p \equiv \beta x^{\prime}+\alpha x
$$

where $\alpha$ and $\beta$ are the Courant-Synder parameters at that location. This means that absent any perturbations, the locus of points in Poincaré space are circles. See Figure 2.

In normalised space, if the position of a particle is at $\left(a_{k}, \phi_{k}\right)$, then the projections onto the axes are

$$
\left.\begin{array}{l}
x_{k}=a_{k} \cos \phi_{k} \\
p_{k}=-a_{k} \sin \phi_{k}=\beta_{k} x_{k}^{\prime}+\alpha_{k} x_{k}
\end{array}\right\}
$$

It follows that the position of this particle at the pickup is

$$
\left.\begin{array}{l}
x_{p}=a_{k}\left(\frac{\beta_{p}}{\beta_{k}}\right)^{\frac{1}{2}} \cos \left(\phi_{k}+\phi_{k p}\right) \\
p_{p}=-a_{k}\left(\frac{\beta_{p}}{\beta_{k}}\right)^{\frac{1}{2}} \sin \left(\phi_{k}+\phi_{k p}\right)
\end{array}\right\}
$$

where the Courant-Synder parameters at the kicker are $\left(\alpha_{k}, \beta_{k}, \gamma_{k}\right)$ and at the pickup are $\left(\alpha_{p}, \beta_{p}, \gamma_{p}\right)$. The phase advance from the kicker to the pickup is $\phi_{k p}$. 


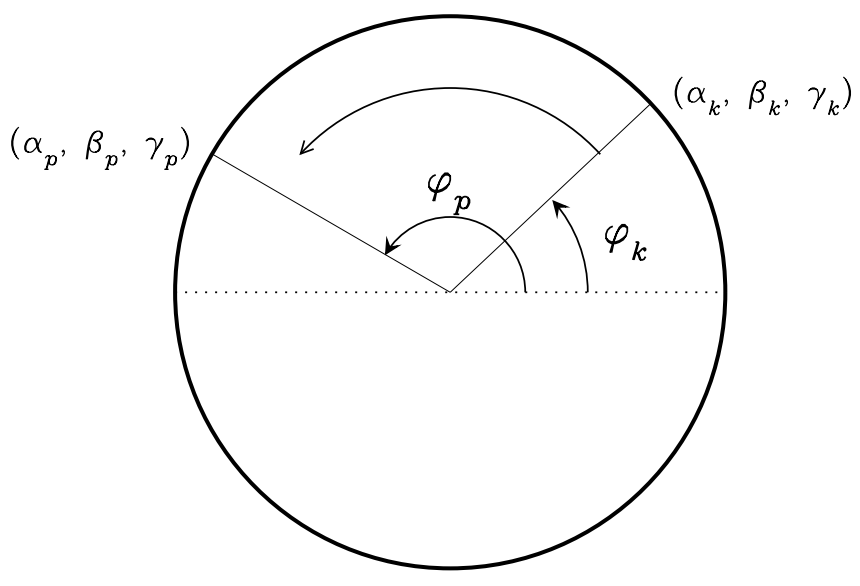

Figure 1 The azimuthal positions of the quadrupole kicker and pickup are $\varphi_{k}$ and $\varphi_{p}$ respectively. The beam is travelling in the counter-clockwise direction from the kicker to the pickup. The Courant-Synder parameters for each location are shown here.

\section{Quadrupole Kicker}

Let us suppose that we have a quadrupole kicker which is a thin lens. The transfer matrix $\boldsymbol{M}_{q}$ is (we will assume that the quadrupole does not create any dispersion)

$$
\boldsymbol{M}_{q}=\left(\begin{array}{cc}
1 & 0 \\
-\frac{1}{f} & 1
\end{array}\right) \equiv\left(\begin{array}{cc}
1 & 0 \\
-F & 1
\end{array}\right)
$$

where $f$ is the focal length of the quadrupole.

Therefore, after the quadrupole kick

$$
\left(\begin{array}{c}
x \\
x^{\prime}
\end{array}\right) \rightarrow\left(\begin{array}{c}
x \\
x^{\prime}-x F
\end{array}\right) \equiv\left(\begin{array}{c}
x \\
x^{\prime}+\Delta x^{\prime}
\end{array}\right)
$$

The perturbative kick only changes the divergence of the beam $x^{\prime}$ and not its position $x$ (See Figure 3) and so from (2), we have the following two linear equations to solve for the 

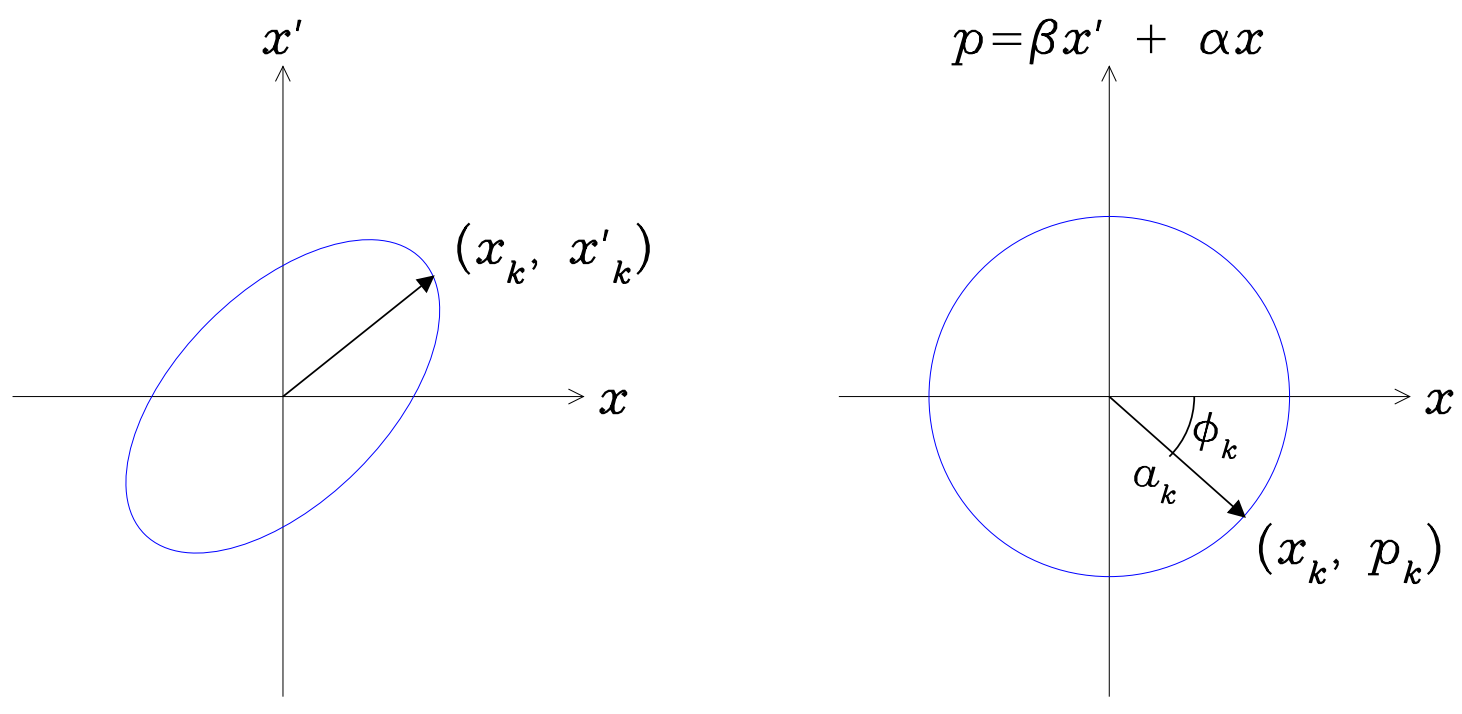

Transform to Normalised Coordinates

Figure 2 The transformation to normalised coordinates maps the phase ellipse to a circle.

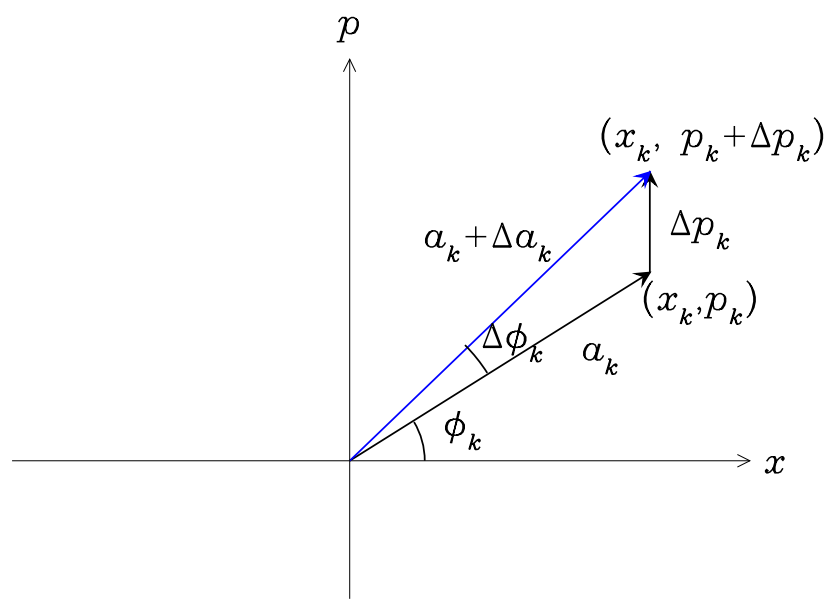

Figure 3 A quadrupole kick changes $p_{k}$ but does not change the position $x_{k}$. The small increase in $p_{k}$ is easily calculated. 
small changes $a_{k} \rightarrow a_{k}+\Delta a_{k}$ and $\phi_{k} \rightarrow \phi_{k}+\Delta \phi_{k}$

$$
\left.\begin{array}{l}
\Delta x_{k}=\Delta a_{k} \cos \phi_{k}-a_{k} \Delta \phi_{k} \sin \phi_{k}=0 \\
\Delta p_{k}=-\left(\Delta a_{k} \sin \phi_{p}+a_{k} \Delta \phi_{k} \cos \phi_{k}\right)=-\beta_{k} a_{k} F_{k} \cos \phi_{k}
\end{array}\right\}
$$

Solving for $\Delta a_{k}$ and $\Delta \phi_{k}$ gives

$$
\left.\begin{array}{rl}
\Delta a_{k} & =\beta_{k} a_{k} F_{k} \cos \phi_{k} \sin \phi_{k} \\
\Delta \phi_{k} & =\beta_{k} F_{k} \cos ^{2} \phi_{k}
\end{array}\right\}
$$

In this method, we will assume that $\left|\beta_{k} F_{k}\right| \ll 1$ and that the betatron tune $Q$ does not change due to this kick. Thus, the position of the particle at turn $n$ is

$$
\left.\begin{array}{l}
a_{k}(n+1)=a_{k}(n)+\frac{1}{2} \beta_{k} a_{k}(n) F_{k}(n) \sin 2 \phi_{k}(n) \\
\phi_{k}(n+1)=\phi_{k}(n)+\beta_{k} F_{k}(n) \cos ^{2} \phi_{k}(n)+2 \pi Q
\end{array}\right\}
$$

Therefore, we can write the change in $a_{k}$ and $\phi_{k}$ per turn in terms of differentials

$$
\left.\begin{array}{rl}
\frac{d a_{k}}{d n} & =\frac{1}{2} \beta_{k} a_{k} F_{k} \sin 2 \phi_{k} \\
\frac{d}{d n}\left(\phi_{k}-2 \pi n Q\right) & =\beta_{k} F_{k} \cos ^{2} \phi_{k}
\end{array}\right\}
$$

The differential equation in $\phi_{k}$ still requires some more manipulation. We define a new angular variable $\psi_{k}=\phi_{k}-n \theta_{Q}-\phi_{k 0}$ where $2 \pi Q=\theta_{Q}$ and $\phi_{k 0}=\phi_{k}(0)$ so that

$$
\left.\begin{array}{rl}
\frac{d a_{k}}{d n} & =\frac{1}{2} \beta_{k} a_{k} F_{k} \sin 2\left(\psi_{k}+n \theta_{Q}+\phi_{k 0}\right) \\
\frac{d \psi_{k}}{d n} & =\beta_{k} F_{k} \cos ^{2}\left(\psi_{k}+n \theta_{Q}+\phi_{k 0}\right)
\end{array}\right\}
$$

which is in a better form for us to solve.

Let us put in an explicit expression for $F_{k}$ (Note: we have chosen the phase of the kick to be zero at $n=0$ because ultimately in a QFR the initial phase of the kick is cancelled out)

$$
F_{k}(n)=\delta F_{k} \sin n \theta_{k} \quad \text { assuming that } \delta F_{k}>0
$$

where $\delta F_{k}$ is the amplitude of the kick satisfying $\left(\epsilon \equiv \beta_{k} \delta F_{k} \ll 1\right)>0$. Substituting this into (10), we have

$$
\left.\begin{array}{rl}
\frac{d a_{k}}{d n} & =\frac{1}{2} \epsilon a_{k} \sin n \theta_{k} \sin 2\left(\psi_{k}+n \theta_{Q}+\phi_{k 0}\right) \\
\frac{d \psi_{k}}{d n} & =\epsilon \sin n \theta_{k} \cos ^{2}\left(\psi_{k}+n \theta_{Q}+\phi_{k 0}\right)
\end{array}\right\}
$$


Notice that $d \psi_{k} / d n$ does not contain $a_{k}$ explicitly and so we will focus our attention on $\psi_{k}$ first.

\section{Solving $\psi_{k}$}

Let us assume that the frequency of the quadrupole kick is about twice the betatron tune $\theta_{Q}$, i.e.

$$
\theta_{k}=2\left(\theta_{Q}-\delta \theta_{k}\right)
$$

where $\delta \theta_{k} / \theta_{Q} \ll 1$. Thus, the rhs of $d \psi_{k} / d n$ in (12) can be expanded with the above and the usual trigonometric functions to give

$$
\begin{aligned}
\frac{d \psi_{k}}{d n}= & \frac{\epsilon}{2} \sin 2 n\left(\theta_{Q}-\delta \theta_{k}\right)+\frac{\epsilon}{4} \sin 2\left(\psi_{k}+n\left(2 \theta_{Q}-\delta \theta_{k}\right)+\phi_{k 0}\right) \\
& -\frac{\epsilon}{4} \sin 2\left(\psi_{k}+n \delta \theta_{k}+\phi_{k 0}\right) \\
= & \text { term oscillating at } 2 \theta_{Q}+\text { term oscillating at } 4\left(\theta_{Q}-\delta \theta_{k}\right)+ \\
& \quad \text { term oscillating at } \delta \theta_{k}
\end{aligned}
$$

We notice that the terms which explicitly contain $2 \theta_{Q}$ and $4 \theta_{Q}$ are highly oscillatory and on average do not contribute significantly to $d \psi_{k} / d n$ (See Numerical Check I). Therefore, we will make the approximation that

$$
\frac{d \psi_{k}}{d n} \approx-\frac{\epsilon}{4} \sin 2\left(\psi_{k}+n \delta \theta_{k}+\phi_{k 0}\right)
$$

This nonlinear differential equation can be solved exactly for the initial condition $\psi_{k}(0)=0$. The solution is

$$
\psi_{k}=-n \delta \theta_{k}-\phi_{k 0}+\tan ^{-1}\left[\frac{1}{4 \delta \theta_{k}}\left(\epsilon+\frac{R\left(\tan \frac{n R}{4}-\chi\right)}{1+\chi \tan \frac{n R}{4}}\right)\right]
$$

where $R=\sqrt{16 \delta \theta_{k}^{2}-\epsilon^{2}}$ and $\chi=\left(\epsilon-4 \delta \theta_{k} \tan \phi_{k 0}\right) / R$. There are two notes: $(i)$ The result of $\tan ^{-1}($.$) is ambiguous up to an integer multiple of \pi$. The choice of this integer 
is to make $\psi_{k}(0)=0$. (ii) $\psi_{k}$ does not go imaginary when $16 \theta_{k}^{2}<\epsilon^{2}$ because

$$
\left.\begin{array}{rl}
R \tan \frac{n R}{4} & =i \operatorname{Im}(R) \times \tan i\left[\frac{n}{4} \operatorname{Im}(R)\right]=i \operatorname{Im}(R) \times i \tanh \left[\frac{n}{4} \operatorname{Im}(R)\right] \\
& =-\operatorname{Im}(R) \times \tanh \left[\frac{n}{4} \operatorname{Im}(R)\right]
\end{array}\right\}
$$

and similarly for $\frac{1}{R} \tan \frac{n R}{4}$.

For completeness, we use the above to expand (16) in powers of $\delta \theta_{k} / \epsilon$ to get

$$
\psi_{k}=-n \delta \theta_{k}-\phi_{k 0}+\tan ^{-1}\left[\frac{\left(1-\tanh \frac{1}{4} n \epsilon\right)}{1+\left(1-\frac{4 \delta \theta_{k}}{\epsilon} \tan \phi_{k 0}\right) \tanh \frac{1}{4} n \epsilon} \tan \phi_{k 0}\right]
$$

and so as $\delta \theta_{k} \rightarrow 0$ and $\epsilon \neq 0, \psi_{k}$ in this limit is

$$
\lim _{\delta \theta_{k} \rightarrow 0} \psi_{k}=-\phi_{k 0}+\tan ^{-1}\left[\frac{1-\tanh \frac{1}{4} n \epsilon}{1+\tanh \frac{1}{4} n \epsilon} \tan \phi_{k 0}\right]
$$

and when $n \rightarrow \infty$

$$
\lim _{\delta \theta_{k} \rightarrow 0, n \rightarrow \infty} \psi_{k}=-\phi_{k 0}
$$

\section{Numerical Check I}

In this section we will perform a numerical check of the approximations that we have derived in the previous section. We will compare the solutions of $\psi_{k}$ obtained from the following:

(i) The difference equations (8) which should give the exact answer for $\psi_{k}$.

(ii) The differential equations (12) which gives a solution of $\psi_{k}$ when $n$ is treated like a continuous variable. ( $\psi_{k}$ is found numerically from (12) with the Runge-Kutta $\operatorname{method}^{7}$ and from Mathematica's NDSolve[].)

(iii) The exact solution of $\psi_{k}$ from (16) of the differential equation (15) where the high frequency terms are neglected. 
The parameters used for our comparisons are shown in Table 1. Note: for simplicity, we have placed the pickup at the same location as the kicker.

\begin{tabular}{c|c|c|c}
\hline \multicolumn{1}{c}{ Table 1. Parameters used in Numerical Check I and II } \\
\hline Parameter & Value & Parameter & Value \\
$\theta_{Q}$ & $2 \pi \times 20.575$ & $\delta \theta_{k}$ & $2 \pi \times\left(10^{-9}, 0.0001,0.01\right)$ \\
$a_{k 0}$ & $1 \mathrm{~mm}$ & $\phi_{k 0}$ & $\pi / 4$ \\
$\phi_{k p}$ & 0 & $\epsilon$ & 0.0001 \\
\hline \hline
\end{tabular}

From Figure 4, we can see that the $\psi_{k}$ approximation (case $($ iii)) from (16) matches the "exact solution" (case $(i)$ )very well in all three cases. It is interesting to note that there are fine structures in the $\delta \theta_{k}=2 \pi \times 0.01$ case from the difference equations (case (i)) which are missing from the solution obtained from the differential equations (12) (case $(i i))$.

\section{Solving for $a_{k}$}

With the $\psi_{k}$ approximation in hand, we can now solve for $a_{k}$ from (12)

$$
\begin{aligned}
\frac{d a_{k}}{d n} & =\frac{1}{2} \epsilon a_{k} \sin 2 n\left(\theta_{Q}-\delta \theta_{k}\right) \sin 2\left(\psi_{k}+n \theta_{Q}+\phi_{k 0}\right) \\
& =\frac{1}{4} \epsilon a_{k}\left[\cos 2\left(n \delta \theta_{k}+\psi_{k}+\phi_{k 0}\right)-\cos 2\left(n\left(2 \theta_{Q}-\delta \theta_{k}\right)+\psi_{k}+\phi_{k 0}\right)\right] \\
& =\text { term oscillating at } 2 \delta \theta_{k}+\text { term oscillating at } 4 \theta_{Q}
\end{aligned}
$$

We proceed in the same manner as in the previous section and note that on average, only the term which is oscillating at $2 \delta \theta_{k}$ contributes to $a_{k}$ and so we will ignore the high frequency term (See Numerical Check II). It is obvious that (21) is integrable because the rhs is separable and thus

$$
a_{k}=\Lambda \exp \left[\frac{1}{4} \epsilon \int d n \cos 2\left(n \delta \theta_{k}+\psi_{k}+\phi_{k 0}\right)\right]
$$



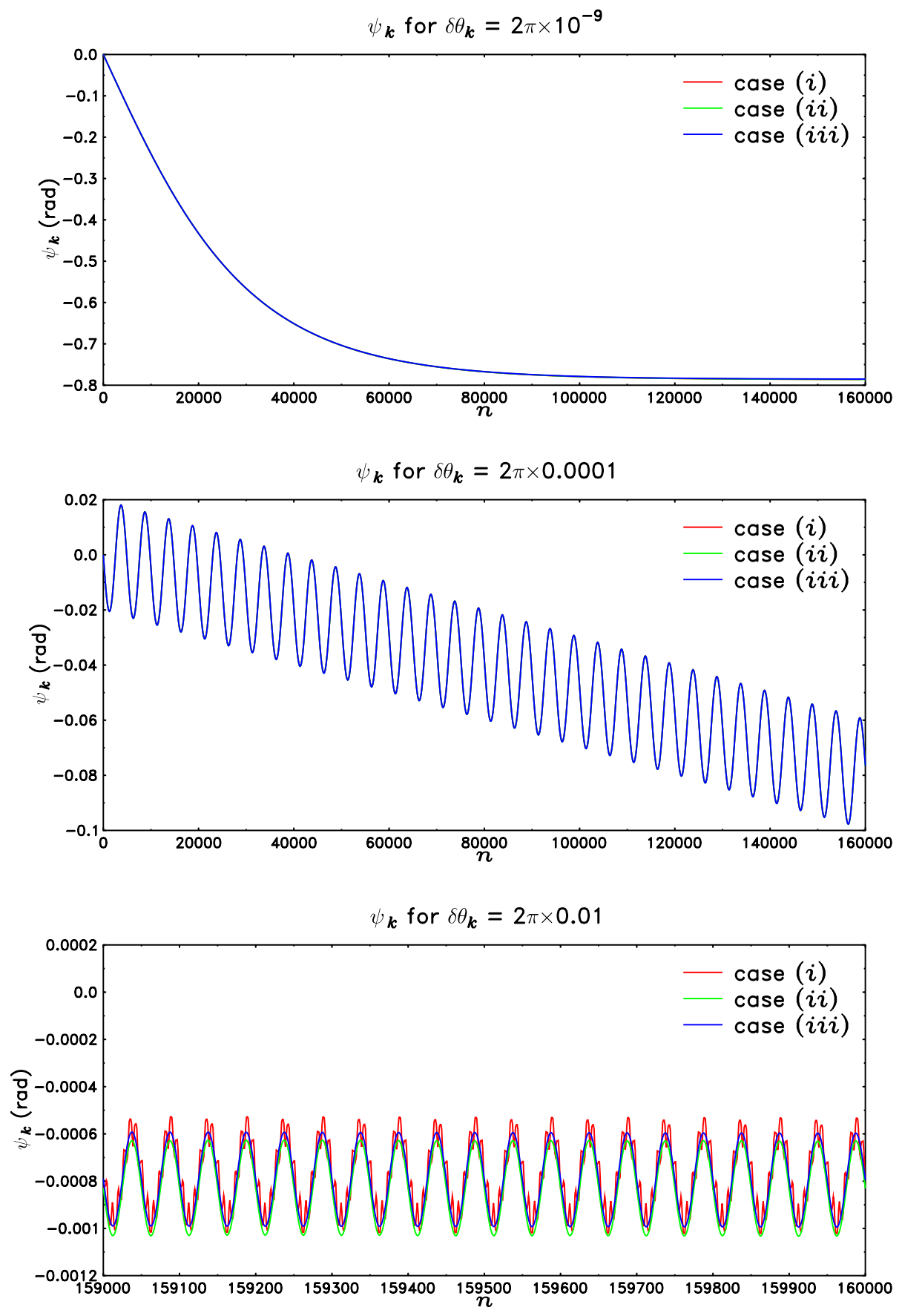

Figure 4 These figures show how well our approximations of $\psi_{k}$ compare to the "exact solution" which is case $(i)$. It is clear that for all three $\delta \theta_{k}$ cases the approximate solution matches the exact solution very well. 
where $\Lambda$ is a constant determined from $a_{k}(0)=a_{k 0}$.

A closer examination of (22) shows that the solution space of $a_{k}$ can be divided into two regions because the integration of (22) depends on the relationship between the two independent parameters $\epsilon$ and $\delta \theta_{k}$. The dividing line between these two regions is when $R \equiv 0$ or

$$
\left|\delta \theta_{k}\right|=\epsilon / 4
$$

See Figure 5.

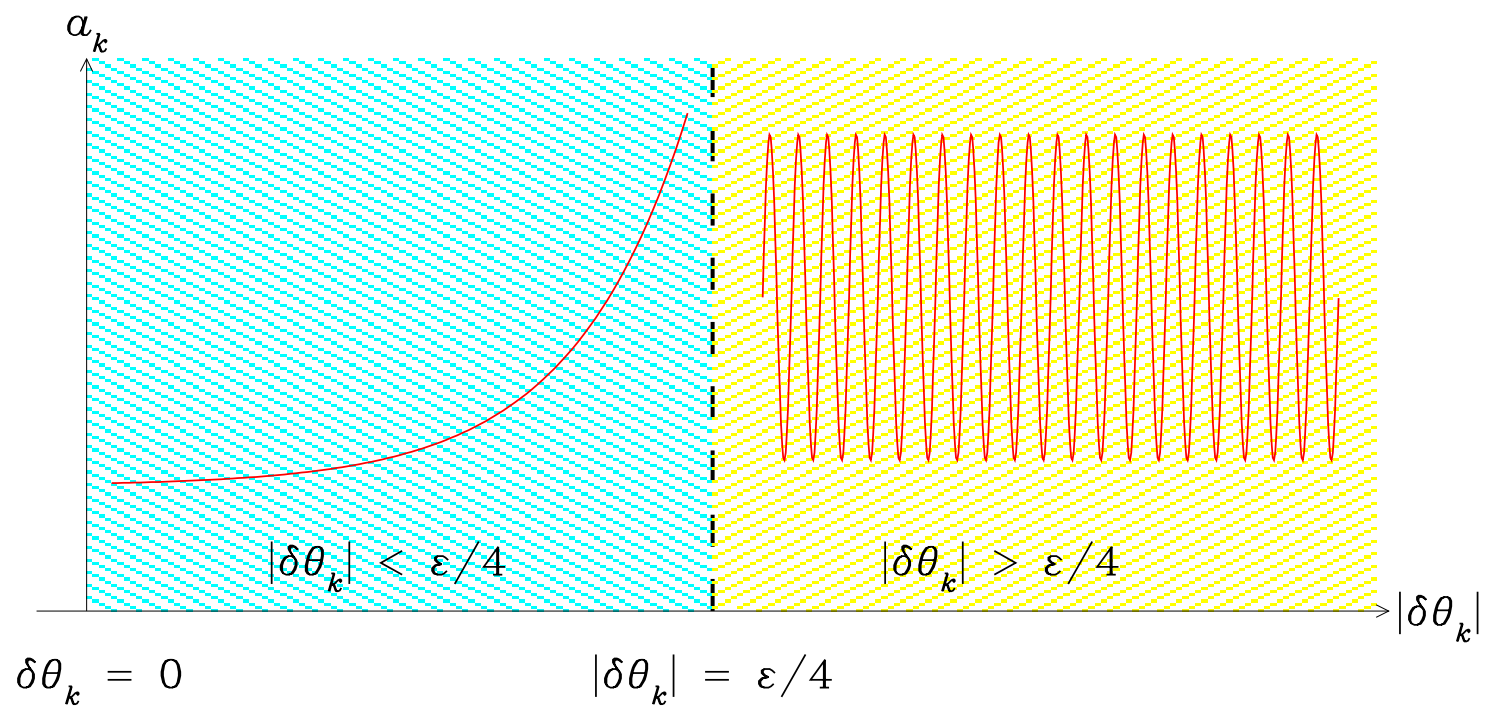

Figure 5 This figure shows how the $a_{k}$ solution space is divided. When $\left|\delta \theta_{k}\right|<\epsilon / 4, a_{k}$ grows exponentially and when $\left|\delta \theta_{k}\right|>\epsilon / 4$, $a_{k}$ oscillates and is bounded. When $\left|\delta \theta_{k}\right|=\epsilon / 4$, we find that $a_{k}$ is constant.

For the case when $\left|\delta \theta_{k}\right|>\epsilon / 4 \neq 0$, if we make the approximation that $\psi_{k}$ is constant because its oscillations are small (see Figure 4(b) and (c)) then (22) is easily integrated

$$
a_{k}=a_{k 0} \exp \left[\frac{\epsilon}{8 \delta \theta_{k}} \sin 2\left(n \delta \theta_{k}+\psi_{k}+\phi_{k 0}\right)\right] \quad \text { for }\left|\delta \theta_{k}\right|>\epsilon / 4
$$


For the case when $0 \leq\left|\delta \theta_{k}\right|<\epsilon / 4$, we note from (16) that

$$
n \delta \theta_{k}+\psi_{k}+\phi_{k 0}=\tan ^{-1}\left[\frac{1}{4 \delta \theta_{k}}\left(\epsilon-\frac{R^{\prime}\left(\tanh \frac{n R^{\prime}}{4}+\chi^{\prime}\right)}{1+\chi^{\prime} \tanh \frac{n R^{\prime}}{4}}\right)\right]
$$

where $R^{\prime}=\sqrt{\epsilon^{2}-16 \delta \theta_{k}^{2}}$ and $\chi^{\prime}=\left(\epsilon-4 \delta \theta_{k} \tan \phi_{k 0}\right) / R^{\prime}$. And as $n \rightarrow \infty$, we find that

$$
n \delta \theta_{k}+\psi_{k}+\phi_{k 0}=\tan ^{-1}\left[\frac{1}{4 \delta \theta_{k}}\left(\epsilon-R^{\prime}\right)\right]=\text { constant }
$$

For a quick check, we see that as $\delta \theta_{k} \rightarrow 0$, the constant is zero, and thus $\psi_{k}=-\phi_{k 0}$ which is exactly the same result as (20).

Therefore, for large $n$, the term $\cos 2\left(n \delta \theta_{k}+\psi_{k}+\phi_{k 0}\right)$ in the integral of (22) is constant and so if we make the approximation that $\cos 2\left(n \delta \theta_{k}+\psi_{k}+\phi_{k 0}\right)$ is constant for all $n$ then (22) is easily integrated to give

$$
a_{k}=a_{k 0} \exp \left\{\frac{1}{4} n \epsilon \cos 2\left(\tan ^{-1}\left[\frac{1}{4 \delta \theta_{k}}\left(\epsilon-R^{\prime}\right)\right]\right)\right\} \quad \text { for } 0 \leq\left|\delta \theta_{k}\right|<\epsilon / 4
$$

There are two interesting notes: $(i)(27)$ is independent of $\phi_{k 0}$ in this approximation which means that in the long time limit the initial phase is not remembered. For example, we can find particle initial conditions which do not behave this way at least for the short term. For example when $\phi_{k 0}=\pi / 2, \delta \theta_{k}=2 \pi \times 10^{-9}$ plus the conditions shown in Table 1 , $a_{k}$ seems to damp for $n<2 \times 10^{5}$ but exponentially blows up as $n \rightarrow \infty$. (ii) the argument of the $\cos ($.$) term is always confined between \pm \pi / 2$ and so this term is never negative and thus $a_{k}$ never damps. See Figure 6.

For the case when $\left|\delta \theta_{k}\right|=\epsilon / 4 \neq 0$, we have $R^{\prime}=0$ and so from (25) we have

$$
n \delta \theta_{k}+\psi_{k}+\phi_{k 0}=\operatorname{sign}\left(\delta \theta_{k}\right) \tan ^{-1}(1)=\operatorname{sign}\left(\delta \theta_{k}\right) \pi / 4
$$

which means that $a_{k}=a_{k 0} \forall n$ because $\cos (2 \times \pm \pi / 4)=0$. 


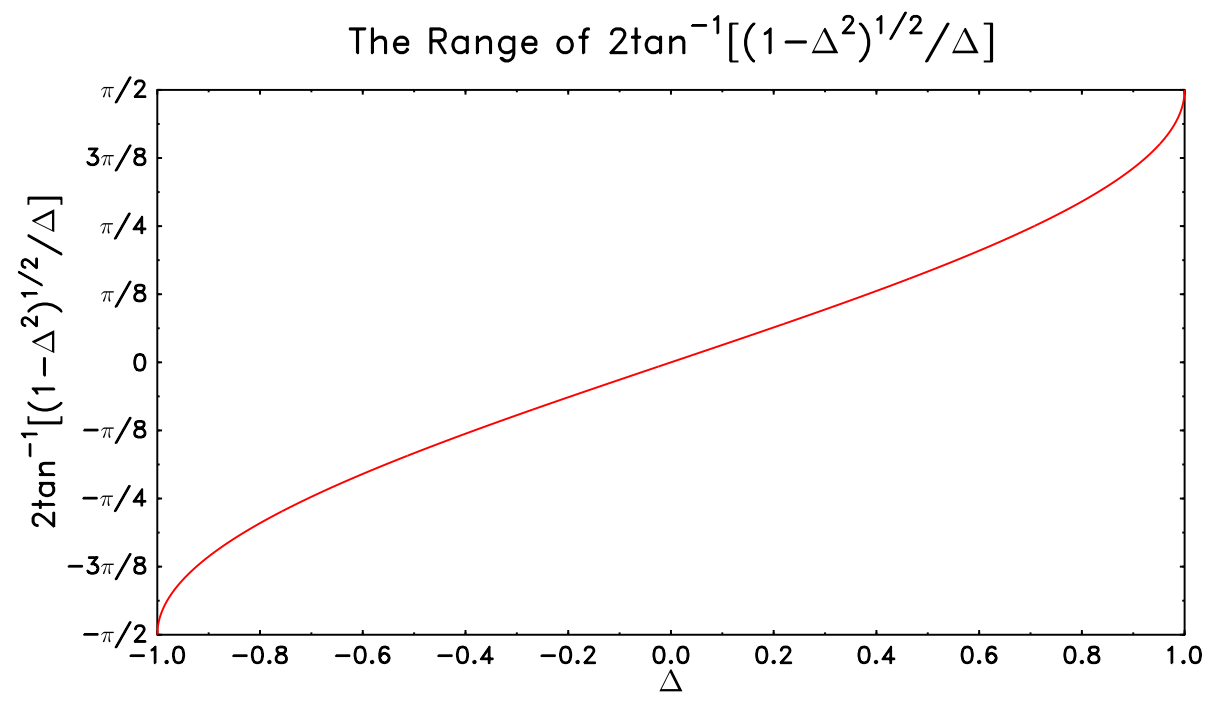

Figure 6 The range of $2 \tan ^{-1}\left[\left(\epsilon-R^{\prime}\right) / 4 \delta \theta_{k}\right]=2 \tan ^{-1}\left[\left(1-\Delta^{2}\right)^{\frac{1}{2}} / \Delta\right]$ where $\Delta=4 \delta \theta_{k} / \epsilon$. It is clear that the range is confined to $\pm \pi / 2$.

\section{Numerical Check II}

First, we use the parameters from Table 1. The exact solution comes from the difference equations (8). The approximate solution used for $\delta \theta_{k}=2 \pi \times 10^{-9}$ come from (27), while $(24)$ is used for $\delta \theta_{k}=2 \pi \times 0.0001$ and $2 \pi \times 0.01$. It is clear from Figure 7 that the exponential growth of $a_{k}$ for $\delta \theta_{k}=2 \pi \times 10^{-9}$ does not start immediately for the "exact solution" but some time later. The difference between the approximate and "exact solution" is rather inconsequential because both blow up exponentially at the same rate. For the $\delta \theta_{k}=2 \pi \times 0.0001$ and $2 \pi \times 0.01$ cases, it is clear that the approximate solutions match well to the "exact solution".

Second, let us use the parameters from Table 2 which show what the solutions look like when $\delta \theta_{k}$ is just a little smaller and larger than $\epsilon / 4=2.5 \times 10^{-5}$, i.e. for $\delta \theta_{k}=$ $\left(\epsilon / 4-0.5 \times 10^{-5}\right)=2 \times 10^{-5}$ and $\delta \theta_{k}=\left(\epsilon / 4+0.5 \times 10^{-5}\right)=3 \times 10^{-5}$. From Figure 8 , we 
can see that the approximate solutions have the same behaviour as the exact solutions.

\begin{tabular}{c|c|c|c}
\hline \multicolumn{1}{c}{ Table 2. Parameters used in Numerical Check II } \\
\hline Parameter & Value & Parameter & Value \\
$\theta_{Q}$ & $2 \pi \times 20.575$ & $\delta \theta_{k}$ & $\epsilon / 4 \pm 0.5 \times 10^{-5}$ \\
$a_{k 0}$ & $1 \mathrm{~mm}$ & $\phi_{k 0}$ & $\pi / 4$ \\
$\phi_{k p}$ & 0 & $\epsilon$ & 0.0001 \\
\hline \hline
\end{tabular}

Image Current

It has been shown elsewhere ${ }^{8}$ that the image current $J$ from a $\delta$-function line current $I$ at $\left(x_{p}, y_{p}\right)$ on the wall of a pickup of radius $b$ is

$$
J=\frac{I\left(r_{p}, \phi_{p}\right)}{2 \pi b} \frac{b^{2}-r_{p}^{2}}{b^{2}+r_{p}^{2}-2 b r_{p} \cos \left(\Phi-\phi_{p}\right)}
$$

where $r_{p}^{2}=x_{p}^{2}+y_{p}^{2}, \tan \phi_{p}=y_{p} / x_{p}$ and $\Phi=0, \pi / 2, \pi$ or $3 \pi / 2$ are the angular positions of the pickup plates.

For a quadrupole pickup (see Figure 9) the image current $J$ on each plate is summed and differenced according to the following equation to yield a quadrupole moment signal $q_{2}$

$$
\left.\begin{array}{rl}
q_{2} & =\frac{(N+S)-(T+B)}{N+S+T+B} \\
& =\frac{2\left(x_{p}^{2}-y_{p}^{2}\right)}{b^{2}\left[1+\left(\frac{x^{2}+y^{2}}{b^{2}}\right)^{2}\right]} \\
& \approx \frac{2}{b^{2}}\left(x_{p}^{2}-y_{p}^{2}\right) \quad \text { if } x_{p}^{2}+y_{p}^{2} \ll b^{2}
\end{array}\right\}
$$

where the labels $(N, S, T, B)$ for the pickup plates are from Miller $^{1}$, and $\Phi=0$ for $N$, $\Phi=\pi$ for $S, \Phi=\pi / 2$ for $T$ and $\Phi=3 \pi / 2$ for $B$

Note: We can compare $q_{2}$ to the quadrupole moment $J_{2}$ of the image current which is 

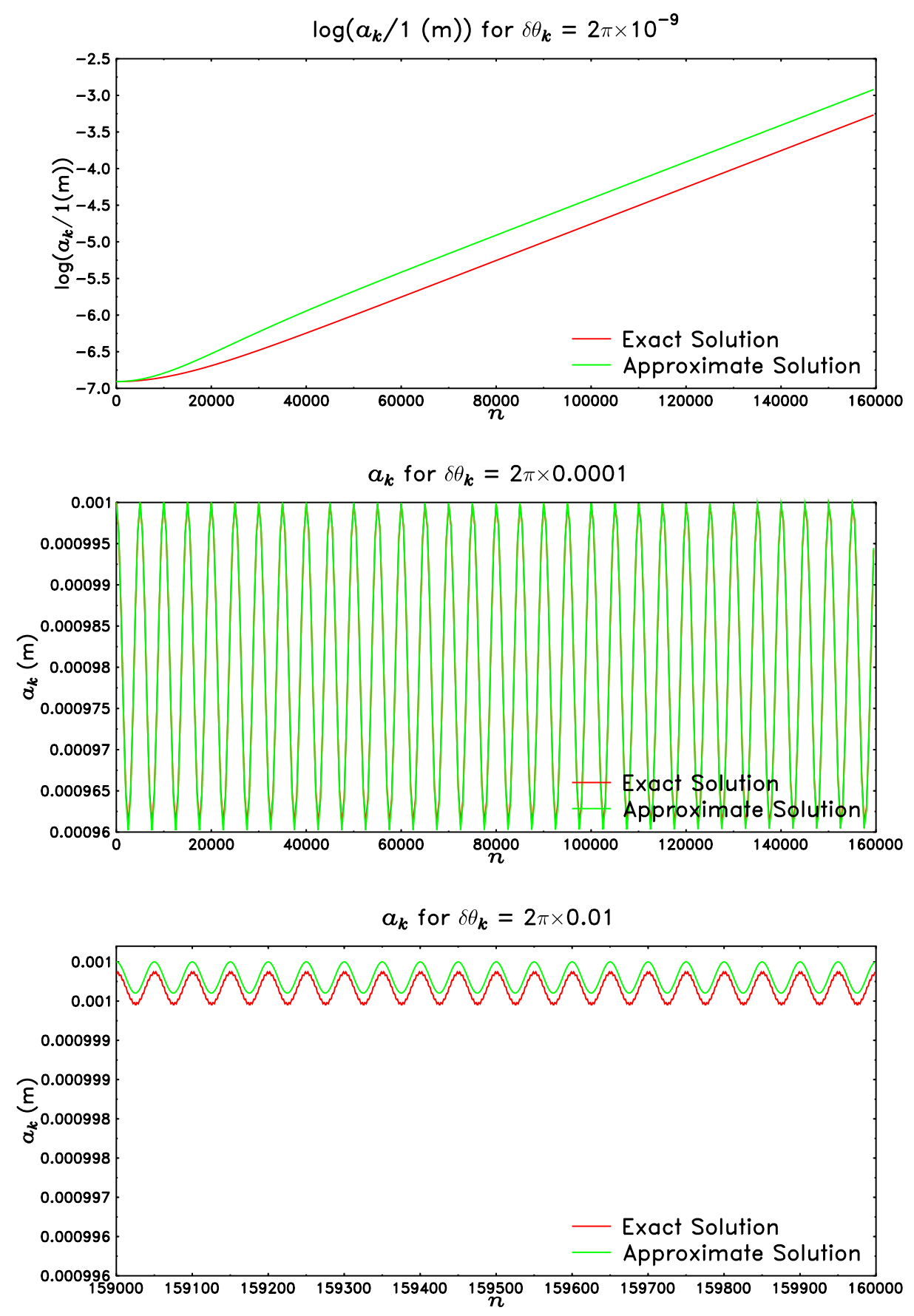

Figure 7 These figures show how well our approximations of $a_{k}$ compare to the "exact solution". Note that in the $\delta \theta_{k}=2 \pi \times 10^{-9}$ case, we have plotted the logarithm of $a_{k}$. In this case (27) is used for $a_{k}$. The other two cases use (24) as solutions. 

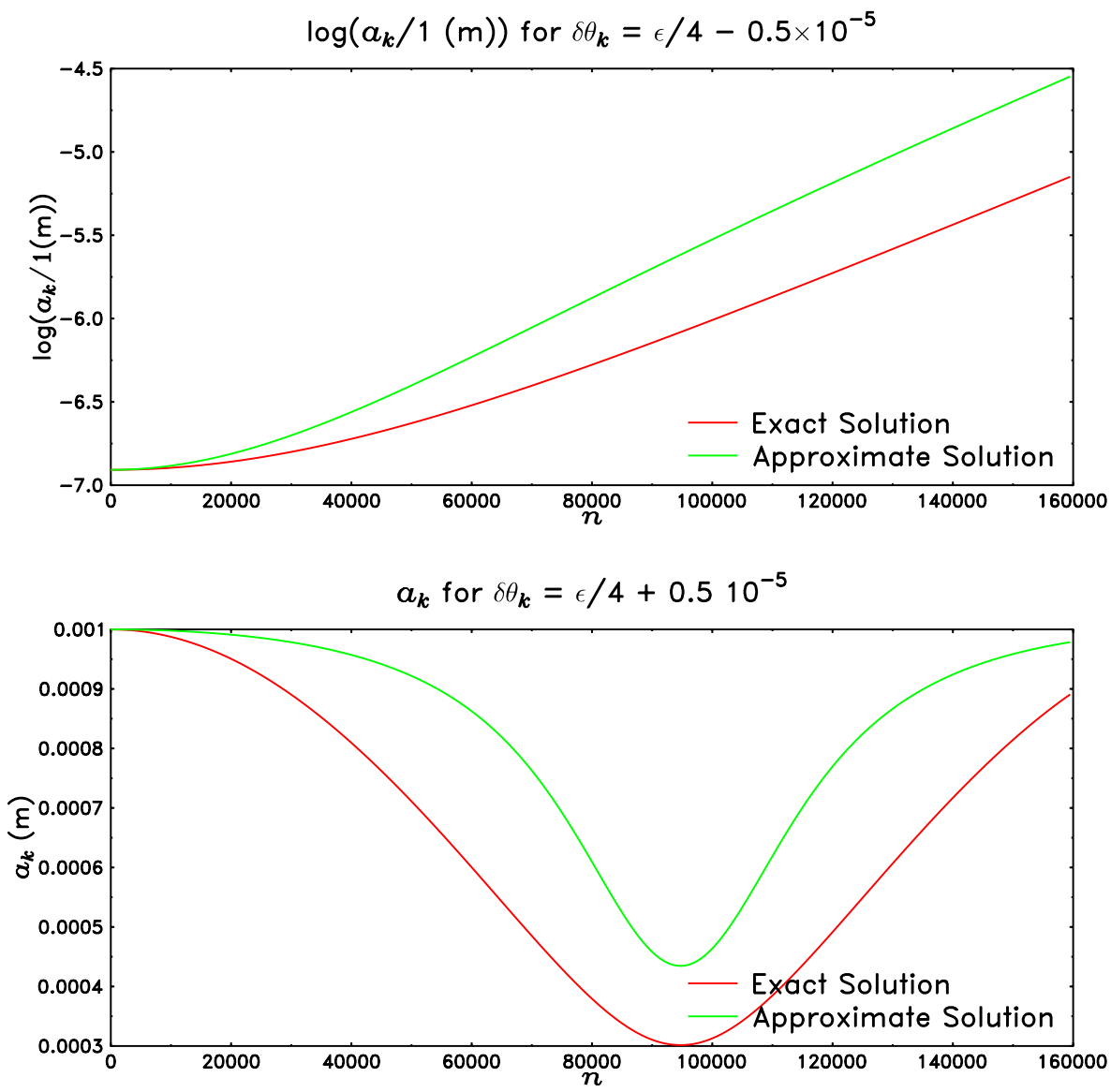

Figure 8 These figures show how $a_{k}$ behaves when $\delta \theta_{k}<\epsilon / 4$ and $\delta \theta_{k}>\epsilon / 4$. When $\delta \theta_{k}<\epsilon / 4, a_{k}$ blows up exponentially while when $\delta \theta_{k}>\epsilon / 4, a_{k}$ remains bounded.

extracted from $(29)$

$$
J_{2}=\frac{I(r, \phi)}{\pi b}\left[\frac{x_{p}^{2}-y_{p}^{2}}{b^{2}} \cos 2 \Phi+2 \frac{x_{p} y_{p}}{b^{2}} \sin 2 \Phi\right]
$$

The form of the $\left(x_{p}^{2}-y_{p}^{2}\right)$ term of $J_{2}$ is clearly captured by $q_{2}$.

The evolution of the transverse position of the particle at $\left(x_{p}, y_{p}\right)$ when there is no coupling between the $x-x_{p}$ and $y$-yp planes comes from (3) and with $a_{k}$ either from (24) 


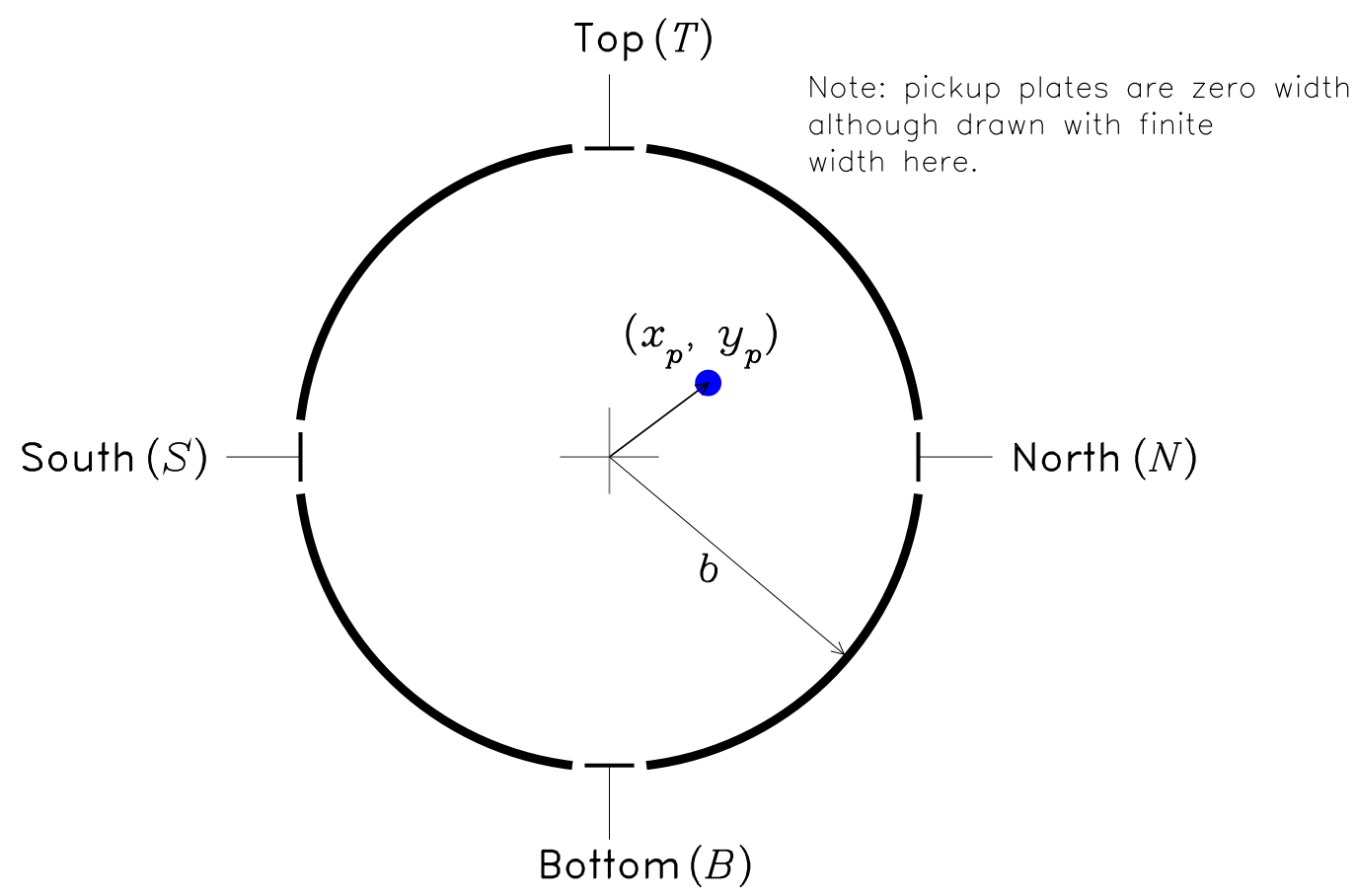

Figure 9 The quadrupole pickup consists of four zero width plates that are summed and differenced appropriately. See (30). The distance of any plate from the centre of the beam pipe is $b$ and the position of the bunch is at $\left(x_{p}, y_{p}\right)$.

or (27) depending on how $\epsilon$ relates to $\delta \theta_{k}$. We can write this down as

$$
\left.\begin{array}{l}
x_{p}(n)=a_{k x}(n)\left(\frac{\beta_{p x}}{\beta_{k x}}\right)^{\frac{1}{2}} \cos \left(n \theta_{Q x}+\psi_{k x}(n)+\phi_{k x 0}+\phi_{k p x}\right) \\
y_{p}(n)=a_{k y}(n)\left(\frac{\beta_{p y}}{\beta_{k y}}\right)^{\frac{1}{2}} \cos \left(n \theta_{Q y}+\psi_{k y}(n)+\phi_{k y 0}+\phi_{k p y}\right)
\end{array}\right\}
$$

where we have added extra subscripts $x$ and $y$ to the notation in order to distinguish between the two planes. Substituting this into (30), we get

$$
\left.\begin{array}{rl}
q_{2}=\frac{1}{b^{2}} & {\left[a_{k x}^{2}(n)\left(\frac{\beta_{p x}}{\beta_{k x}}\right) \cos 2\left(n \theta_{Q x}+\psi_{k x}(n)+\phi_{k x 0}+\phi_{k p x}\right)\right.} \\
- & a_{k y}^{2}(n)\left(\frac{\beta_{p y}}{\beta_{k y}}\right) \cos 2\left(n \theta_{Q y}+\psi_{k y}(n)+\phi_{k y 0}+\phi_{k p y}\right) \\
& \left.+a_{k x}^{2}(n)\left(\frac{\beta_{p x}}{\beta_{k x}}\right)-a_{k y}^{2}(n)\left(\frac{\beta_{p y}}{\beta k y}\right)\right]
\end{array}\right\}
$$




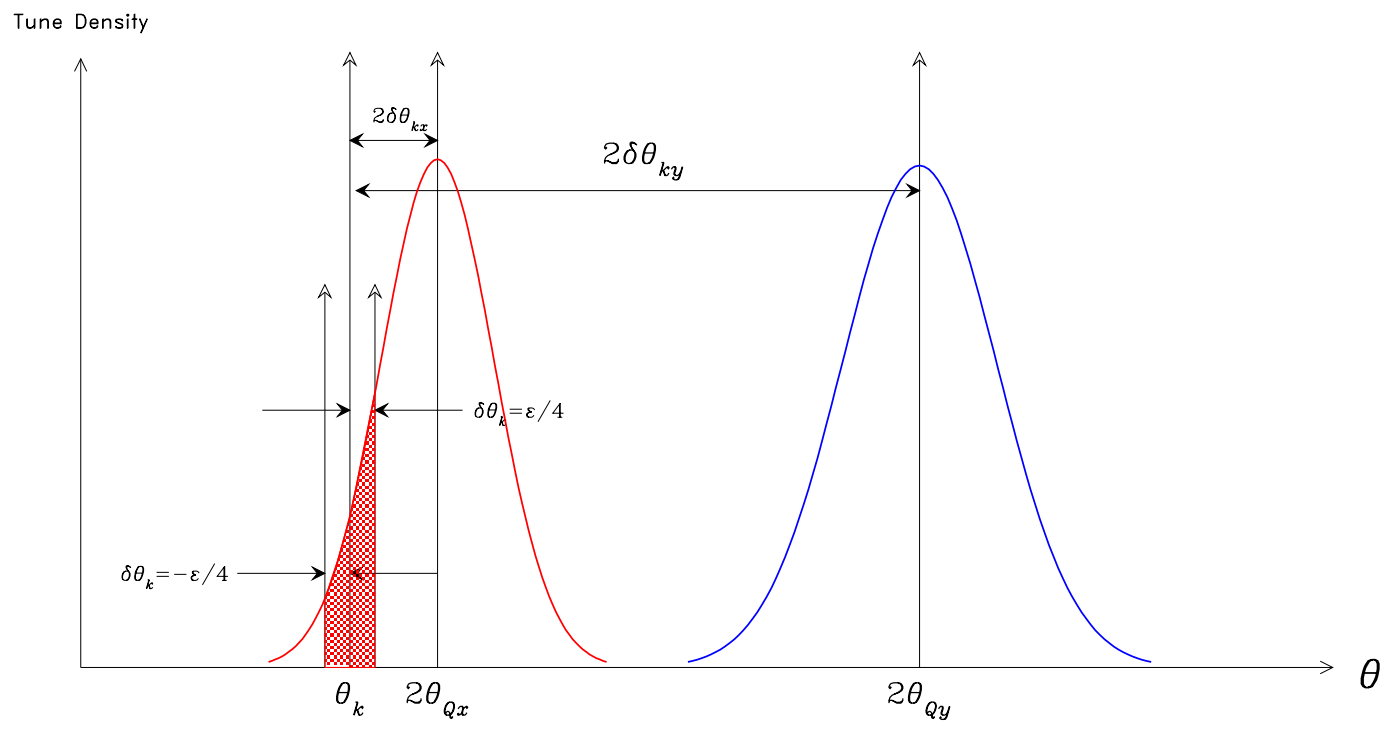

Figure 10 The kick at $\theta_{k}$ excites the $x-x_{p}$ plane quadrupole tune distribution and is far from the $y$ - $y_{p}$ quadrupole tune distribution. Note that within the region shaded in red, the kicker excites the distribution strongly. In fact the particles here will blow up exponentially.

For the QFR, we are only interested in terms which are oscillating at the kicker frequency $\theta_{k}=2\left(\theta_{Q x}-\delta \theta_{k x}\right)=2\left(\theta_{Q y}-\delta \theta_{k y}\right)$ and so we will only keep terms which are oscillating at this frequency to yield

$$
\left.\begin{array}{rl}
q_{2}= & \frac{a_{k x}^{2}(n)}{b^{2}}\left(\frac{\beta_{p x}}{\beta_{k x}}\right) \times \\
& \cos 2\left(n\left(\theta_{Q x}-\delta \theta_{k x}\right)+\tan ^{-1}\left[\frac{1}{4 \delta \theta_{k x}}\left(\epsilon_{x}+\frac{R_{x}\left(\tan \frac{n R_{x}}{4}-\chi_{x}\right)}{1+\chi_{x} \tan \frac{n R_{x}}{4}}\right)\right]+\phi_{k p x}\right) \\
& -\frac{a_{k y}^{2}(n)}{b^{2}}\left(\frac{\beta_{p y}}{\beta_{k y}}\right) \times \\
& \cos 2\left(n\left(\theta_{Q y}-\delta \theta_{k y}\right)+\tan ^{-1}\left[\frac{1}{4 \delta \theta_{k y}}\left(\epsilon_{y}+\frac{R_{y}\left(\tan \frac{n R_{y}}{4}-\chi_{y}\right)}{1+\chi_{y} \tan \frac{n R_{y}}{4}}\right)\right]+\phi_{k p y}\right)
\end{array}\right\}
$$

where we have used (16). 
Let us choose the $x-x_{p}$ plane to be the plane of interest. If the vertical betatron tune $Q y$ is far from the horizontal tune $Q x$, then $\left|\epsilon_{y} / 4 \delta \theta_{k y}\right| \approx 0$. (See Figure 10). Substituting this into (24) and (16), we have $a_{k y} \approx a_{k y 0}$ and $\psi_{k y} \approx 0$. Thus (34) can be simplified to give

$$
\left.\begin{array}{rl}
q_{2}= & \frac{a_{k x}^{2}(n)}{b^{2}}\left(\frac{\beta_{p x}}{\beta_{k x}}\right) \times \\
& \cos 2\left(n\left(\theta_{Q x}-\delta \theta_{k x}\right)+\tan ^{-1}\left[\frac{1}{4 \delta \theta_{k x}}\left(\epsilon_{x}+\frac{R_{x}\left(\tan \frac{n R_{x}}{4}-\chi_{x}\right)}{1+\chi_{x} \tan \frac{n R_{x}}{4}}\right)\right]+\phi_{k p x}\right) \\
& \quad-\frac{a_{k y 0}^{2}}{b^{2}}\left(\frac{\beta_{p y}}{\beta_{k y}}\right) \cos 2\left(n\left(\theta_{Q y}-\delta \theta_{k y}\right)+\phi_{k y 0}+\phi_{k p y}\right)
\end{array}\right\}
$$

From Figure 10, we notice that when the kick frequency is within the quadrupole tune distribution, the particles in the shaded red region will eventually blow up exponentially in time. This means that this method will cause emittance growth which unfortunately cannot be stopped with Landau damping.

\section{No Landau Damping}

It is clear from (27) and (35) that $a_{k}$ will blow up exponentially when the kick is within the quadrupole tune distribution. Unfortunately, the $a_{k}$ solution does not have any poles (i.e. $\delta \theta_{k}=0$ ) in the denominator of (27) and thus the usual method of introducing Landau damping by integrating over the quadrupole distribution of the bunch is not applicable here (See, for example, $\left.\mathrm{Chao}^{9}\right)$. In fact, we cannot stop the exponential blow up of the bunch with Landau damping.

\section{Quadrupole Frequency Response}

Since we cannot rely on Landau damping to stop the exponential growth of the bunch, 
technically the frequency response does not exist because it does not converge as $t \rightarrow \infty$. To get out of this conundrum, we will instead, assume that the kick duration $\Delta T$ is very short so that the blow up is minimal and we will calculate the frequency response at the end of this time.

To get some sense of the length of $\Delta T$, we suppose that the frequency resolution required is $0.0001 f_{\mathrm{rev}}$ ( $f_{\mathrm{rev}}$ is the revolution frequency) and thus the number of turns required for this resolution is $1 / 0.0001=10^{4}$. We can throw in 10 averages and so the number of turns become $10^{5}$. The relative growth of $a_{k}$ is calculated by using the numbers in Appendix II for $\delta \theta_{k}=0$ and $\epsilon=4 \times 10^{-7}$

$$
\frac{a_{k}}{a_{k 0}}=\exp \left(\frac{1}{4} \times 10^{5} \times 4 \times 10^{-7}\right)=1.01
$$

which means that there is a $1 \%$ growth of $a_{k}$ which is not insignificant. However, for only one measurement we can assume that $a_{k}$ is constant for a kick of duration $10^{5}$ turns (approximately $2.1 \mathrm{~s}$ for the Tevatron). With this assumption (35) becomes

$$
\left.\begin{array}{rl}
q_{2}= & \frac{a_{k x 0}^{2}}{b^{2}}\left(\frac{\beta_{p x}}{\beta_{k x}}\right) \times \\
& \cos 2\left(n\left(\theta_{Q x}-\delta \theta_{k x}\right)+\tan ^{-1}\left[\frac{1}{4 \delta \theta_{k x}}\left(\epsilon_{x}+\frac{R_{x}\left(\tan \frac{n R_{x}}{4}-\chi_{x}\right)}{\left.1+\chi_{x} \tan \frac{n R_{x}}{4}\right)}+\phi_{k p x}\right)\right.\right. \\
& \quad-\frac{a_{k y 0}^{2}}{b^{2}}\left(\frac{\beta_{p y}}{\beta_{k y}}\right) \cos 2\left(n\left(\theta_{Q y}-\delta \theta_{k y}\right)+\phi_{k y 0}+\phi_{k p y}\right)
\end{array}\right\}
$$

Next, if we assume that $10^{5}$ turns is approximately equivalent to $n \rightarrow \infty$, then

$$
\begin{aligned}
q_{2}= & \frac{a_{k x 0}^{2}}{b^{2}}\left(\frac{\beta_{p x}}{\beta_{k x}}\right) \times \\
& \cos 2\left(n\left(\theta_{Q x}-\delta \theta_{k x}\right)+\tan ^{-1}\left[\frac{1}{4 \delta \theta_{k x}}\left(\epsilon_{x}-R_{x}^{\prime}\right)\right]+\phi_{k p x}\right) \\
& -\frac{a_{k y 0}^{2}}{b^{2}}\left(\frac{\beta_{p y}}{\beta_{k y}}\right) \cos 2\left(n\left(\theta_{Q y}-\delta \theta_{k y}\right)+\phi_{k y 0}+\phi_{k p y}\right)
\end{aligned}
$$

where we have used (26) and assumed that $0 \leq\left|\delta \theta_{k x}\right|<\epsilon_{x} / 4$. 


\section{Many Particles}

Up to this point we have been working with a single particle with initial conditions $\left(a_{k x 0}, \phi_{k x 0}\right)$ and $\left(a_{k y 0}, \phi_{k y 0}\right)$. We will now calculate the quadrupole moment for a distribution of particles in transverse phase space. Let us assume that in normalised phase space the distributions in $\left(x, p_{x}\right)$ and $\left(y, p_{y}\right)$ are stationary. Let us suppose that $\theta_{k}$ is within the quadrupole tune distribution of the $x-x_{p}$ distribution but is far away from the $y-y_{p}$ distribution. See Figure 10. We notice that since $\theta_{k}$ is far away from $2 \theta_{Q y}$, this means that there are hardly any particles that will oscillate at $\theta_{k}$ and so the $a_{k y 0}$ term of (38) is approximately zero when integrated over the $y$-yp quadrupole tune density. Therefore, we will drop this term. Thus (38) becomes

$$
d q_{2}=\frac{a_{k x 0}^{2}}{b^{2}}\left(\frac{\beta_{p x}}{\beta_{k x}}\right) \cos 2\left(n\left(\theta_{Q x}-\delta \theta_{k x}\right)+\tan ^{-1}\left[\frac{1}{4 \delta \theta_{k x}}\left(\epsilon_{x}-R_{x}^{\prime}\right)\right]+\phi_{k p x}\right)
$$

where $d q_{2}$ is the contribution to the quadrupole moment from one particle with initial conditions $\left(a_{k x 0}, \phi_{k x 0}\right)$.

For the QFR, we have to change our perspective to that of the kicker. Looking at Figure 11, we see that

$$
\left.\begin{array}{l}
\theta_{k}=2 \theta_{Q}-\delta \theta_{Q} \quad \text { in kicker perspective } \\
\theta_{k}=2\left(\theta_{Q}-\delta \theta_{k}\right) \quad \text { by definition }
\end{array}\right\}
$$

where $\delta \theta_{Q}$ is the new variable which brings us to the perspective of the kicker. Equating the two equations in (40), we have $\delta \theta_{k}=\frac{1}{2} \delta \theta_{Q}$. Therefore, (39) in this new perspective becomes

$$
d q_{2}=\frac{a_{k 0}^{2}}{b^{2}}\left(\frac{\beta_{p}}{\beta_{k}}\right) \cos \left(n \theta_{k}+2 \tan ^{-1}\left[\frac{1}{2 \delta \theta_{Q}}\left(\epsilon-R^{\prime}\right)\right]+2 \phi_{k p}\right)
$$

We have also dropped " $x$ " from the subscripts because it is obvious that we are only looking at the $x-x^{\prime}$ plane only. So, given the kicker tune, a particle which has a quadrupole tune that is $\delta \theta_{Q}$ away from $\theta_{k}$ will evolve according to (41). 
Now, we have to sum in the contribution from particles which have initial condition $\left(a_{k 0}, \phi_{k 0}\right)$ but have different quadrupole tunes. In order to make this solvable, we will make the approximation that only particles which have quadrupole tunes which are in the red region of Figure 10 contribute to the quadrupole moment when the kick tune is $\theta_{k}$. The justification for this approximation comes from Figure 5 where it is clear that the particles which will eventually dominate the quadrupole moment are those which satisfy $\left|\delta \theta_{k}\right|<\epsilon / 4$ or $\left|\delta \theta_{Q}\right|<\epsilon / 2$.

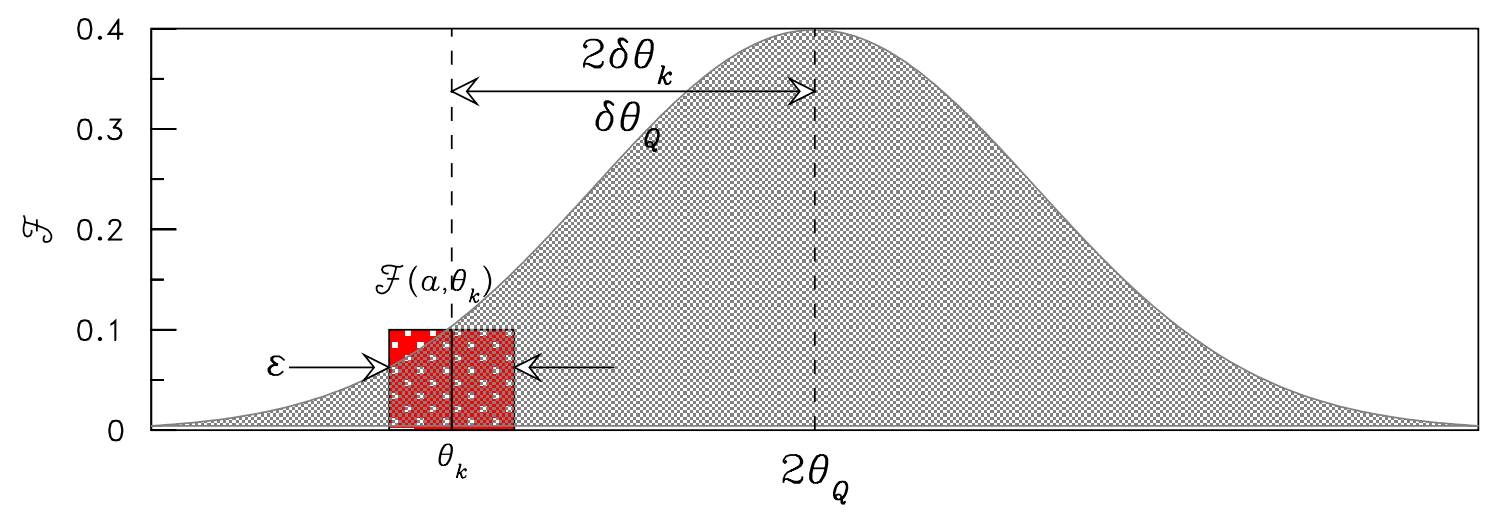

Figure 11 For each $a$ there is an $\mathcal{F}$ which tells us what fraction of the particles have that quadrupole tune. Clearly, the area under the curve must be 1 . The area shaded in red has a width of $\epsilon$ and height $\mathcal{F}\left(a, \theta_{k}\right)$ which approximates the fraction of particles which will contribute to the quadrupole moment at quadrupole tune $\theta_{k}$.

We note from (20) that the $\tan ^{-1}[$.$] term in (41) is zero when \delta \theta_{Q}=0$ and so if we make the approximation that the $\tan ^{-1}[$.$] term for all the particles in the red region is also$ zero, we can sum them up to give

$$
\left.\begin{array}{rl}
d\left\langle q_{2}\right\rangle & =\int_{-\epsilon / 4}^{\epsilon / 4} \mathcal{F}\left(a_{k 0}, \theta_{k}-\theta\right) d \theta \times \frac{a_{k 0}^{2}}{b^{2}}\left(\frac{\beta_{p}}{\beta_{k}}\right) \cos \left(n \theta_{k}+2 \phi_{k p}\right) \\
& \approx \epsilon \mathcal{F}\left(a_{k 0}, \theta_{k}\right) \times \frac{a_{k 0}^{2}}{b^{2}}\left(\frac{\beta_{p}}{\beta_{k}}\right) \cos \left(n \theta_{k}+2 \phi_{k p}\right)
\end{array}\right\}
$$

where $\mathcal{F}(a, \theta)$ is the quadrupole tune density function for particles at radius $a$. See Figure 11 . 
$d\left\langle q_{2}\right\rangle$ gives us the contribution to the quadrupole moment from some fraction of particles on the circle $a_{k 0}$ in $(a, \phi)$ space and quadrupole tune $\theta_{k}$. If we are given the particle density function $\mathcal{A}$ in $(a, \phi)$ space, then the "number" of particles (because everything is normalized to 1 , this "number" is really the fraction of particles) which lie on the circle $a_{k 0}$ is simply $2 \pi a_{k 0} \mathcal{A}\left(a_{k 0}\right) d a_{k 0}$. ( $\mathcal{A}$ is independent of $\phi$ because we have assumed that the phase space density is stationary). And thus the contribution to the quadrupole moment from particles on circles with radii from 0 to $\infty$ which have quadrupole tune $\theta_{k}$ is simply ${ }^{\dagger}$

$$
\left.\begin{array}{rl}
\left\langle q_{2}\right\rangle & =\int_{0}^{\infty} d a 2 \pi a \mathcal{A}(a) \times \epsilon \mathcal{F}\left(a, \theta_{k}\right) \frac{a^{2}}{b^{2}}\left(\frac{\beta_{p}}{\beta_{k}}\right) \cos \left(n \theta_{k}+2 \phi_{k p}\right) \\
& =\epsilon \frac{2 \pi}{b^{2}}\left(\frac{\beta_{p}}{\beta_{k}}\right) \int_{0}^{\infty} d a a^{3} \mathcal{F}\left(a, \theta_{k}\right) \mathcal{A}(a) \times \cos \left(n \theta_{k}+2 \phi_{k p}\right)
\end{array}\right\}
$$

The quadrupole frequency response $\mathcal{H}$ is easily read off from (43) when we recall from (10) that the quadrupole kick is $\epsilon \sin \left(n \theta_{k}\right)$ and so we have

$$
\left.\begin{array}{rl}
\text { magnitude response }|\mathcal{H}| & =\frac{2 \pi}{b^{2}}\left(\frac{\beta_{p}}{\beta_{k}}\right) \int_{0}^{\infty} d a a^{3} \mathcal{F}\left(a, \theta_{k}\right) \mathcal{A}(a) \\
\text { phase response } \arg (\mathcal{H}) & =\frac{1}{2} \pi+2 \phi_{k p}\left(\theta_{k}\right)
\end{array}\right\}
$$

where we have reminded ourselves that the phase advance from the kicker to the pickup $\phi_{k p}$ is a function of the betatron tune.

\section{Calculating the Emittance}

To obtain a specific solution for the emittance from (44), we have to specify the distribution $\mathcal{A}$. If we assume that the distribution of particles projected onto the $x$-axis at the kicker is Gaussian with standard deviation $\sigma_{k}$, then the distribution projected onto the $p$-axis must also be Gaussian with standard deviation $\sigma_{k}$ because we have assumed that

$\dagger$ Technically, the integral should be confined to within the beam pipe of radius $b$. However, the limit can be taken to $\infty$ because of the approximation that we have made in (30). 
the phase space distribution is stationary. Thus, $\mathcal{A}$ is a bi-Gaussian

$$
\mathcal{A} d x d p=\frac{1}{2 \pi \sigma_{k}^{2}} \exp \left[-\frac{x^{2}+p^{2}}{\left(2 \sigma_{k}\right)^{2}}\right] d x d p \quad \text { and } \quad \int_{-\infty}^{\infty} d x d p \mathcal{A}=1
$$

And in polar coordinates $(a, \phi)$, we have

$$
\mathcal{A} a d a d \phi=\frac{1}{2 \pi \sigma_{k}^{2}} \exp \left[-\frac{a^{2}}{\left(2 \sigma_{k}\right)^{2}}\right] a d a d \phi
$$

We also know from the definition of $\mathcal{F}$ (see Figure 11), that

$$
\int_{0}^{\infty} d \theta_{k} \mathcal{F}\left(a, \theta_{k}\right)=1
$$

and so from the magnitude response we find that when we integrate $|\mathcal{H}|$ over $\theta_{k}$, we can factor out the $\mathcal{F}$ dependence and thus

$$
\int_{0}^{\infty} d \theta_{k}|\mathcal{H}|=\frac{2 \pi}{b^{2}}\left(\frac{\beta_{p}}{\beta_{k}}\right) \int_{0}^{\infty} d a a^{3} \mathcal{A}(a) \equiv Q_{2}
$$

We note that we can explicitly obtain the solution to the integral in (48) with $\mathcal{A}$ defined in $(46)$, i.e.

$$
\int_{0}^{\infty} d a a^{3} \mathcal{A}(a)=\frac{4 \sigma_{k}^{2}}{\pi}
$$

which we can then substitute into (48) to get

$$
\left.\begin{array}{rl}
Q_{2} & =\frac{8 \sigma_{k}^{2}}{b^{2}}\left(\frac{\beta_{p}}{\beta_{k}}\right) \\
\sigma_{k}^{2} & =\frac{1}{8} Q_{2} b^{2}\left(\frac{\beta_{k}}{\beta_{p}}\right)
\end{array}\right\}
$$

Finally, by using the $95 \%$ emittance formula for a Gaussian bunch we can obtain the emittance $\varepsilon$

$$
\varepsilon=\frac{6 \pi \sigma_{k}^{2}}{\beta_{k}}=\frac{3 \pi b^{2}}{4 \beta_{p}} Q_{2}
$$

We summarise the approximations and assumptions which we have made to obtain the above: 
(i) The kick duration is short so that $a_{k}$ is approximately constant.

(ii) The beam is well decoupled and the $y$ quadrupole tune is far away from the $x$ quadrupole tune.

(iii) Only a small region $\left|\delta \theta_{Q}\right|<\epsilon / 2$ around $\theta_{k}$ in the quadrupole tune distribution contributes to the frequency response.

(iv) The phase space distribution $\mathcal{A}$ is stationary and is bi-Gaussian with standard deviation $\sigma_{k}$.

\section{Numerical Check IIIa}

We can check our results of the previous section by numerically calculating the QFR for known beam distributions in both normalised phase space and betatron tune. From the integral of the magnitude of the QFR we can calculate the standard deviation by using (50) and then compare it with the input standard deviation. Here are the details of the numerical check:

(i) Create an $M$ particle bi-Gaussian distribution in normalised phase space with standard deviation $\sigma_{k}$ and with a Gaussian betatron tune distribution with standard deviation $\sigma_{Q}$ and mean $\theta_{Q}$.

(ii) Kick the beam with a chirp. This type of kick is defined to be

$$
\operatorname{chirp}(n)=\epsilon \sin n \theta_{k}=\epsilon \sin \left(n \times 2 \times 2 \pi Q_{k}(n)\right) \quad \text { where } \quad Q_{k}(n)=Q_{\text {start }}+\frac{d Q_{k}}{d n} n
$$

and $Q_{\text {start }}$ is the starting betatron kick tune. For the simulation, we have made $Q_{k}$ increase linearly w.r.t. $n$, i.e. if the chirp sweeps from $Q_{\text {start }}$ to $Q_{\text {stop }}$ in $N$ turns then we can write $d Q_{k} / d n=\left(Q_{\text {stop }}-Q_{\text {start }}\right) / N$. $Q_{\text {start }}$ and $Q_{\text {stop }}$ have been chosen so that they symmetrically enclose $\theta_{Q} / \pi$. See Figure 12 . 
(iii) For each particle in the distribution, we calculate its quadrupole moment $q_{2}$ using (30) at every turn when it is kicked with the chirp. We have placed the quadrupole kicker and pickup at the same location so that the phase advance between the kicker and the pickup is zero. We calculate $\left\langle q_{2}\right\rangle$ by averaging the contribution to $q_{2}$ from each of the $M$ particles from each turn.

(iv) The QFR is calculated after $N$ turns using

$$
\mathrm{QFR}=\frac{\operatorname{FFT}\left[\left\langle q_{2}(n)\right\rangle\right]}{\operatorname{FFT}[\operatorname{chirp}(n)]}
$$

where FFT[.] is the $N$ turn Fast Fourier transform. An average of $N_{A}$ QFR's is used smooth out the QFR.

$(v)$ We notice that the QFR magnitude has a small DC offset which contributes to the integral if not corrected. The source of this offset is from the division of a small number by another small number (which should have been zero!). For example, from Figure 12(b), for a perfect chirp, the magnitude of the chirp at say, $\theta / 2 \pi=0.12$ should be zero - this means that the QFR is undefined here. Instead, we have some erroneous response here from the "leakage" of the chirp because clearly from the quadrupole magnitude (Figure 12(a)), it is close to zero here $\left(\sim 10^{-8}\right)$, i.e. not kicked. To correct for this we fit the QFR to a Lorentzian

$$
g(\theta)=\frac{a_{1}}{\left(\theta-a_{2}\right)^{2}+a_{3}^{2}}+a_{4}
$$

where $a_{1}, a_{2}, a_{3}$ and $a_{4}$ are free parameters and then ignore $a_{4}$ when we perform the integral. $g(\theta)$ sans $a_{4}$ is the corrected QFR magnitude. From Figure 12(c), we can see that the fit to the QFR magnitude with $g(\theta)$ is excellent.

(vi) The integral of the QFR magnitude is calculated by using the corrected QFR which is discussed above. The infinite integral of a Lorentzian is

$$
\int_{-\infty}^{\infty} \frac{a_{1} d \theta}{\left(\theta-a_{2}\right)^{2}+a_{3}^{2}}=\pi\left(\frac{a_{1}}{a_{3}}\right)=Q_{2}
$$


(vii) $\sigma_{k}$ is found by substituting in $Q_{2}$ from (55) into (50). This is then compared with the $\sigma_{k}$ used to create the original distribution.

\begin{tabular}{c|c|c|c}
\hline \multicolumn{2}{c}{ Table 3. Parameters used in Numerical Check IIIa } \\
\hline $\begin{array}{c}\text { Parameter } \\
\theta_{Q}\end{array}$ & Value & Parameter & Value \\
$\epsilon$ & $2 \pi \times 20.575$ & $N$ & 8192 \\
$b$ & $1.5 \times 10^{-4}$ & $N_{A}$ & 10 \\
$\beta_{p}=\beta_{k}$ & $10 \mathrm{~m}$ & $M$ & $20 \times 10^{3}, 40 \times 10^{3}$ \\
\hline \hline
\end{tabular}

The simulations that we have done can be divided into two: one set where we use $20 \times 10^{3}$ particles and the other $40 \times 10^{3}$ particles. For each $\sigma_{k}$, we also vary $\sigma_{Q}$ by $2 \pi \times(0.00025), 2 \pi \times(0.0005)$, and $2 \pi \times(0.001)$ to generate the input distribution. The rest of the simulation parameters are shown in Table 8. We calculate $\sigma_{k}$ from the QFR of each particle distribution using the procedure outlined above. Figure 13 shows the results. It is clear from the simulations that $\sigma_{k}$ (calculated) is linearly related to $\sigma_{k}$ (input) and as expected from the theory, also independent of $M, \sigma_{Q}$. To show that the results are independent of $\epsilon$, we calculated a few points (shown as purple $\times$ 's in Figure 13) for $\epsilon=5 \times 10^{-4}, \sigma_{Q}=2 \pi \times 0.00025$ and $M=40 \times 10^{3}$.

For the parameters of Table 8 , the linear fits of $\sigma_{k}$ (calculated) to $\sigma_{k}$ (input) are

$$
\begin{aligned}
& \sigma_{k}(\text { calculated })=(1.002 \pm 0.006) \sigma_{k}\left(\text { input }, M=20 \times 10^{3}\right) \\
& \sigma_{k}(\text { calculated })=(1.000 \pm 0.005) \sigma_{k}\left(\text { input }, M=40 \times 10^{3}\right) \\
& \sigma_{k}(\text { calculated })=(0.995 \pm 0.004) \sigma_{k}\left(\text { input }, \text { combined } M=20 \times 10^{3} \text { and } 40 \times 10^{3}\right)
\end{aligned}
$$

These fits are extremely good and show that numerical solution agrees with the analytic solution very well. 

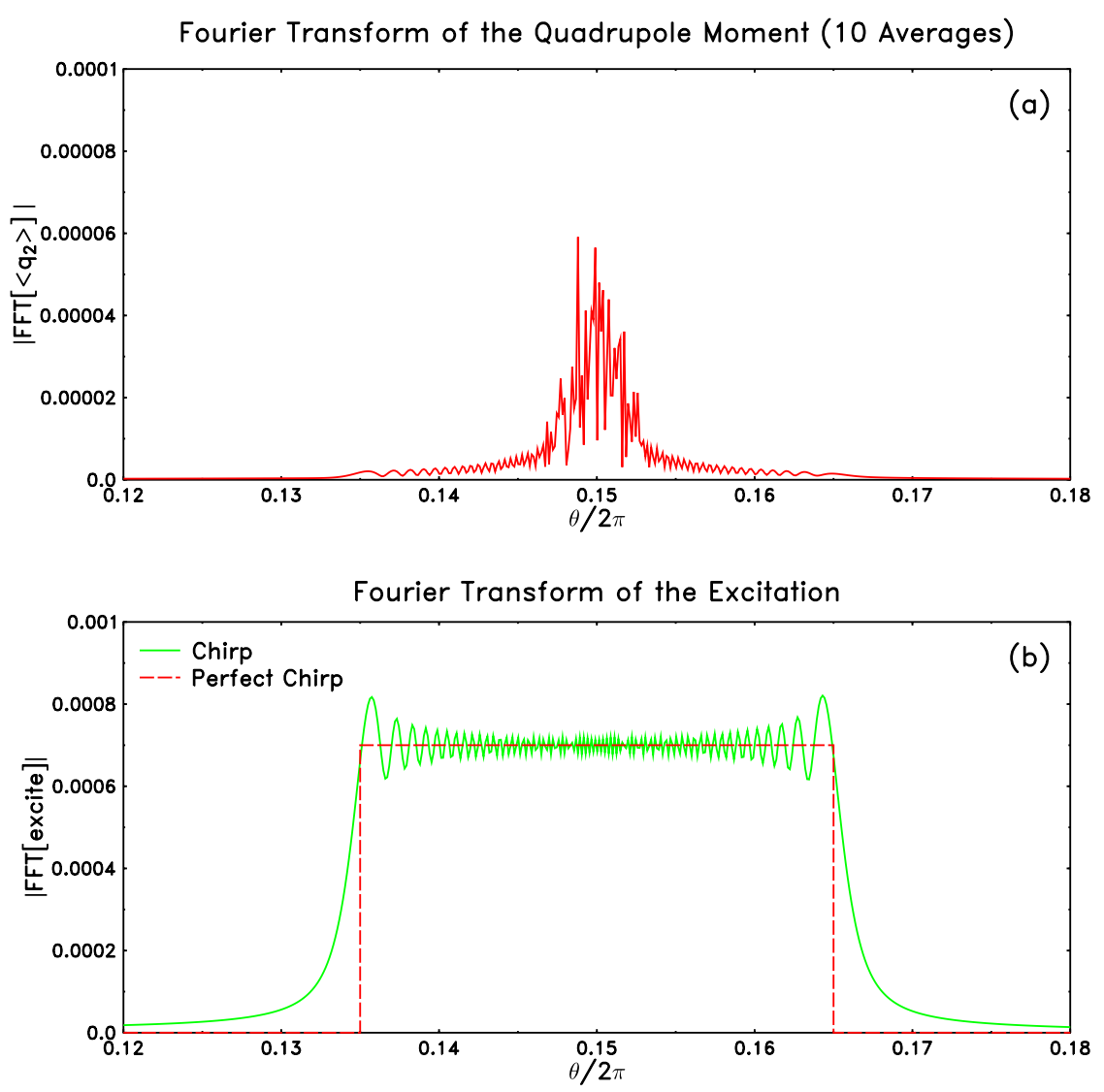

The Magnitude of the QFR

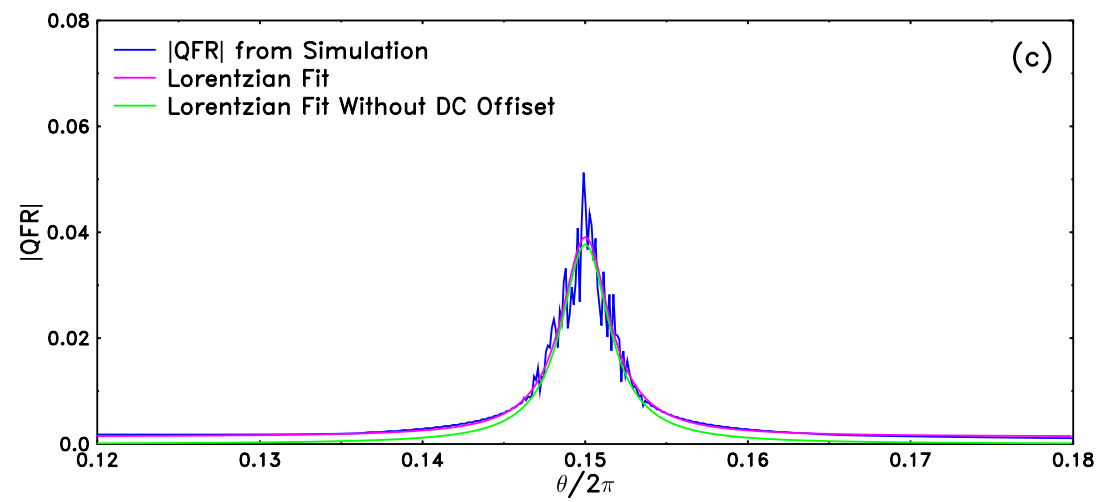

Figure 12 These figures show how the $|\mathrm{QFR}|$ is numerically calculated from the FFT[excite] and FFT[chirp]. In this example $\sigma_{k}=$ $0.5 \mathrm{~mm}, \sigma_{Q}=2 \pi \times 0.0005, \epsilon=2.5 \times 10^{-4}$ and $M=40 \times 10^{3}$. Note: The chirp excitation is well centred about $2 Q_{k}$ when $Q_{\text {start }}$ and $Q_{\text {stop }}$ are not symmetric about $Q_{k}$. In this example $Q_{k}=20.575$, $Q_{\text {start }}=(20.575-4 \times 0.002)$ and $Q_{\text {stop }}=20.575=Q_{k}$. 


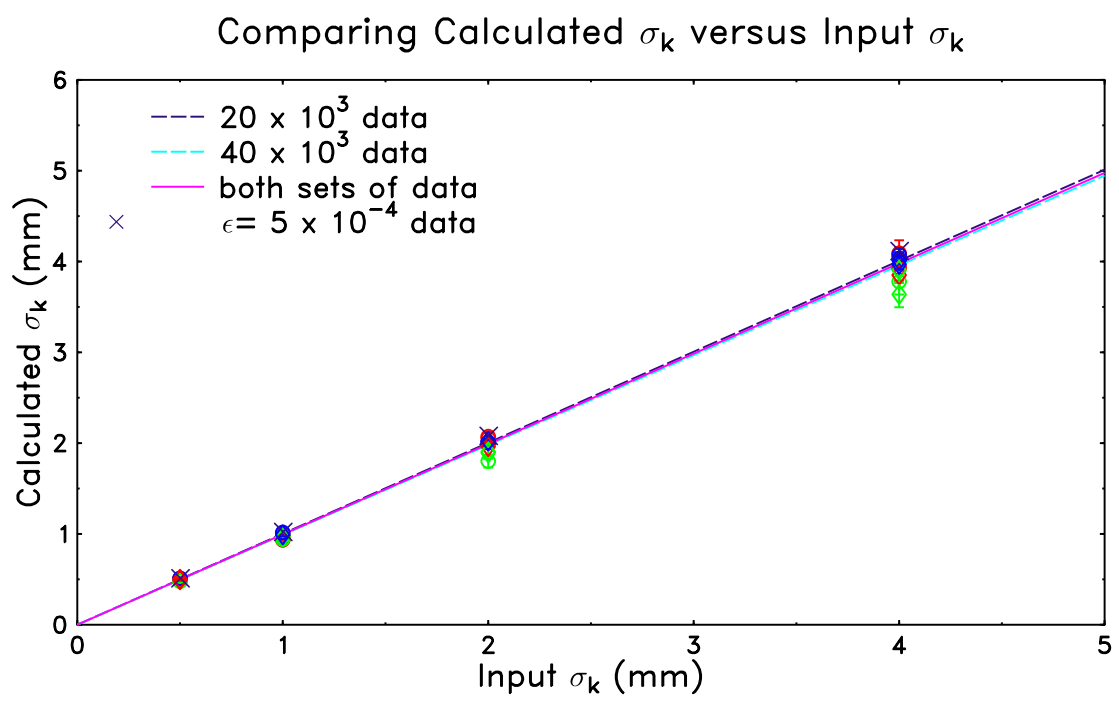

Figure 13 This graph compares the $\sigma_{k}$ used to create the normalised phase space and the calculated $\sigma_{k}$ from the QFR. The colours and shapes of the markers corresponds to different conditions used in forming the initial distribution. See Table 4 for the keys needed for deciphering these markers.

\begin{tabular}{c|c|c|c}
\hline \multicolumn{2}{c}{ Table 4. Keys for Deciphering the Markers in Figure 13 } \\
\hline Num. Particles & Shape & $\boldsymbol{\sigma}_{\boldsymbol{Q}}$ & Colour \\
$20 \times 10^{3}$ & $\bigcirc$ & $2 \pi \times 0.0025$ & blue \\
$40 \times 10^{3}$ & $\diamond$ & $2 \pi \times 0.005$ & red \\
- & - & $2 \pi \times 0.01$ & green \\
\hline
\end{tabular}

Numerical Check IIIb

We want to verify that the emittance does grow when the beam is kicked continuously. From the Theory section, we recall that for a given maximum kick size $\epsilon$, and quadrupole kick tune $\theta_{k}$, particles within the region $\left|\delta \theta_{Q}\right|<\epsilon / 2$ around $\theta_{k}$ will blow up exponentially. 
For example, if we use the numbers in Table 3 , when $\epsilon=2.5 \times 10^{-4}$, the particles in the region $\pm 1.25 \times 10^{-4}$ around $\theta_{k}=2 \times 2 \pi \times 20.575$ will blow up. Let us simulate this with the distribution which has $\sigma_{k}=0.5 \mathrm{~mm}, \sigma_{Q}=2 \pi \times 2.5 \times 10^{-4}$ and $\theta_{Q}=2 \pi \times 20.575$ for 81920 turns (which corresponds to $1.7 \mathrm{~s}$ in the Tevatron). The result is shown in Figure 14(a) which clearly shows that are particles which are greater than $4 \sigma_{k}=2 \mathrm{~mm}$ from the centre of the bunch. In fact $3 \%$ of the total particles are in the halo.

In section Numerical Check IIIa, we have minimised the growth problem by using a chirp instead of a series of pure sine waves when we measure the QFR. From the numbers that we have used to create Figure $12, d Q_{k} / d n=9.8 \times 10^{-7}$, and so particles which have quadrupole tunes which are $\sim \epsilon$ around $\theta_{k}$ gets kicked about $\epsilon / d Q_{k} / d n=2.5 \times 10^{-4} / 9.8 \times$ $10^{-7}=255$ times per QFR measurement. The simulation with the same initial parameters as the above but chirped 10 times (1.7 $\mathrm{s}$ in the Tevatron) does not show any growth. See Figure 14(b). However, with 100 chirps (17 s in the Tevatron), the number of kicks on the particles at each $Q_{k}$ is about 2500 and a halo of particles (about $2 \%$ of the total particles) is produced. See Figure 14(c).

For completeness, we have also simulated the case when $\epsilon=4 \times 10^{-7}$ which corresponds to the value of $\epsilon$ found in Appendix I for a realistic quadrupole kicker. In this case, because the kick is much smaller, the betatron tune distribution which we have used is $\sigma_{Q}=2 \pi \times 10^{-8}$. After $8192 \times 10^{3}$ turns (which corresponds to $172 \mathrm{~s}$ or $3 \mathrm{~min}$ in the Tevatron), the normalised phase space also gets distorted. See Figure 14(d). 

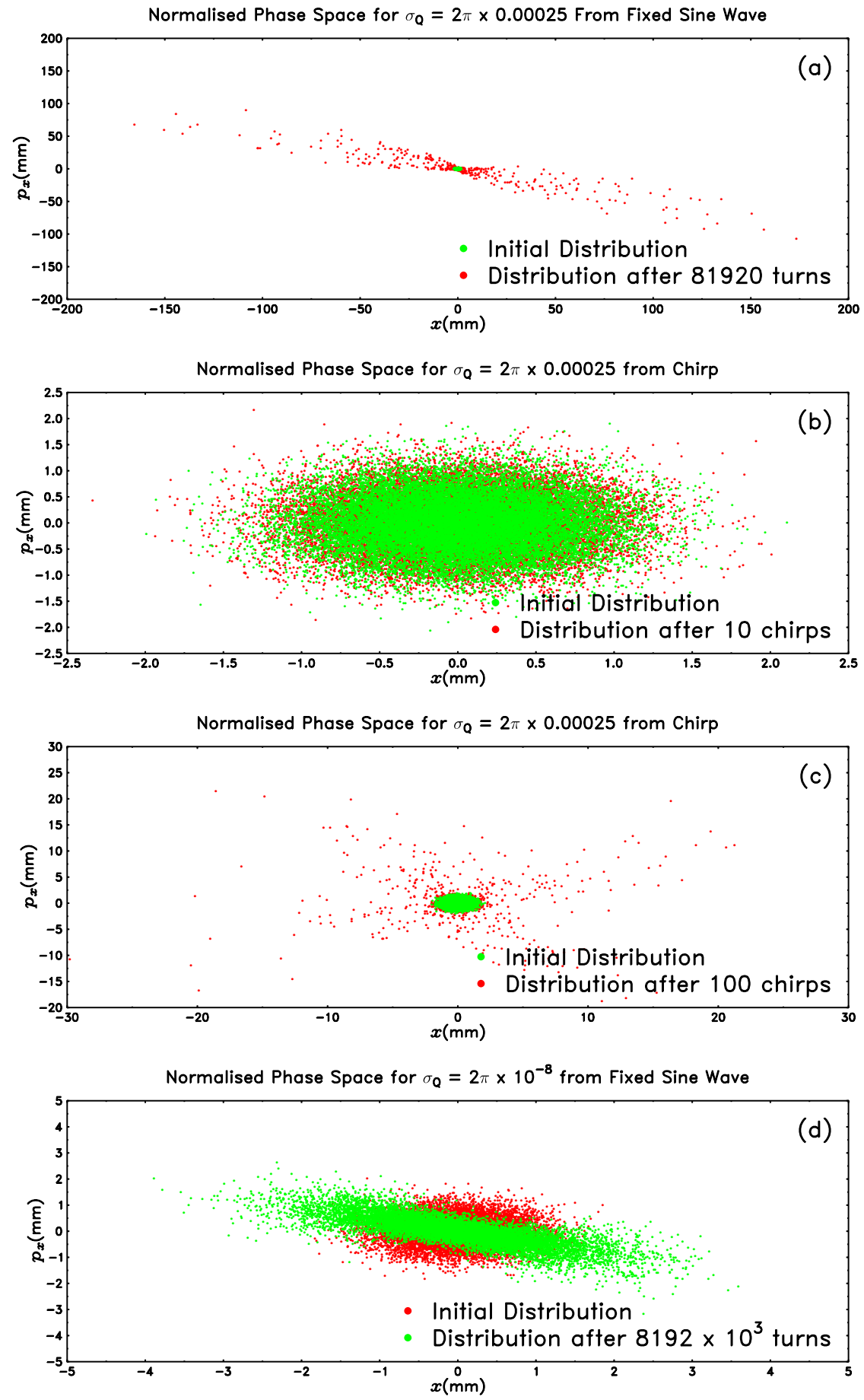

Figure 14 For $\epsilon=2.5 \times 10^{-4}$, for both a fixed sine wave or chirp, the emittance will grow if applied for a sufficiently long time. For $\epsilon=4 \times 10^{-7}$, the emittance gets distorted after $8192 \times 10^{3}$ turns. 


\section{BUNCHED BEAM}

Up to this point, we have been working with coasting beam. For bunched beam, we have to include the effects of the RF which introduces synchrotron motion which affects the azimuthal position of the bunch. If we suppose the bunch is extremely short then we

can think of it as a $\delta$-function bunch. ${ }^{\ddagger}$ Its azimuthal position at any time $t$ at the kicker is

$$
\varphi_{k}=\omega_{\mathrm{rev}} t+\Delta \phi_{s} \cos \left(\Omega_{s} t+\phi_{s 0}\right)
$$

where $\omega_{\text {rev }}$ is the angular revolution frequency of the synchronous particle, $\Omega_{s}$ is the synchrotron frequency, $\left(\Delta \phi_{s}, \phi_{s 0}\right)$ are the polar coordinates of the bunch in longitudinal phase space $(\kappa \Delta E / E, \Delta \varphi)$ where $\kappa=\sqrt{\eta h E / 2 \pi(v / c)^{2} q V}, \eta$ is the slip factor, $h$ is the harmonic number, $\Delta E / E$ is the relative energy w.r.t. synchronous particle, $v / c$ is the relative velocity w.r.t. speed of light, $q$ is the electronic charge, $V$ is the peak RF voltage and $\Delta \varphi$ is the phase w.r.t. the synchronous particle. See Figure 15. Differentiating $\varphi_{k}$ w.r.t. $t$, we get

$$
\left.\begin{array}{rl}
\dot{\varphi}_{k} & =\omega_{\mathrm{rev}}-\Omega_{s} \Delta \phi_{s} \sin \left(\Omega_{s} t+\phi_{s 0}\right) \\
& \equiv \omega_{\mathrm{rev}}+\Delta \omega_{\mathrm{rev}}
\end{array}\right\}
$$

The relative revolution frequency change $\Delta \omega_{\text {rev }} / \omega_{\text {rev }}$ is proportional to $\Delta p / p$. The proportionality constant is the slip factor $-\eta$ and so

$$
\left.\begin{array}{rl}
\frac{\Delta \omega_{\mathrm{rev}}}{\omega_{\mathrm{rev}}} & =-\eta \frac{\Delta p}{p} \\
\Rightarrow \quad \frac{\Delta p}{p} & =+\frac{1}{\eta} \frac{\Omega_{s}}{\omega_{\mathrm{rev}}} \Delta \phi_{s} \sin \left(\Omega_{s} t+\phi_{s 0}\right)
\end{array}\right\}
$$

after substituting in $\Delta \omega_{\text {rev }}$ from $(58)$.

If our particle has a different momentum than the synchronous particle, i.e. it has a

$\ddagger$ Some of the mathematics in this section is very similar to that used by McGinnis where he derived the expressions required for calculating chromaticity from phase demodulation. However, in his derivation he used $\varphi_{k}=\omega_{\text {rev }} t+\Delta \phi_{s} \sin \left(\Omega_{s} t+\phi_{s 0}\right)$. See Ref. 10 . 


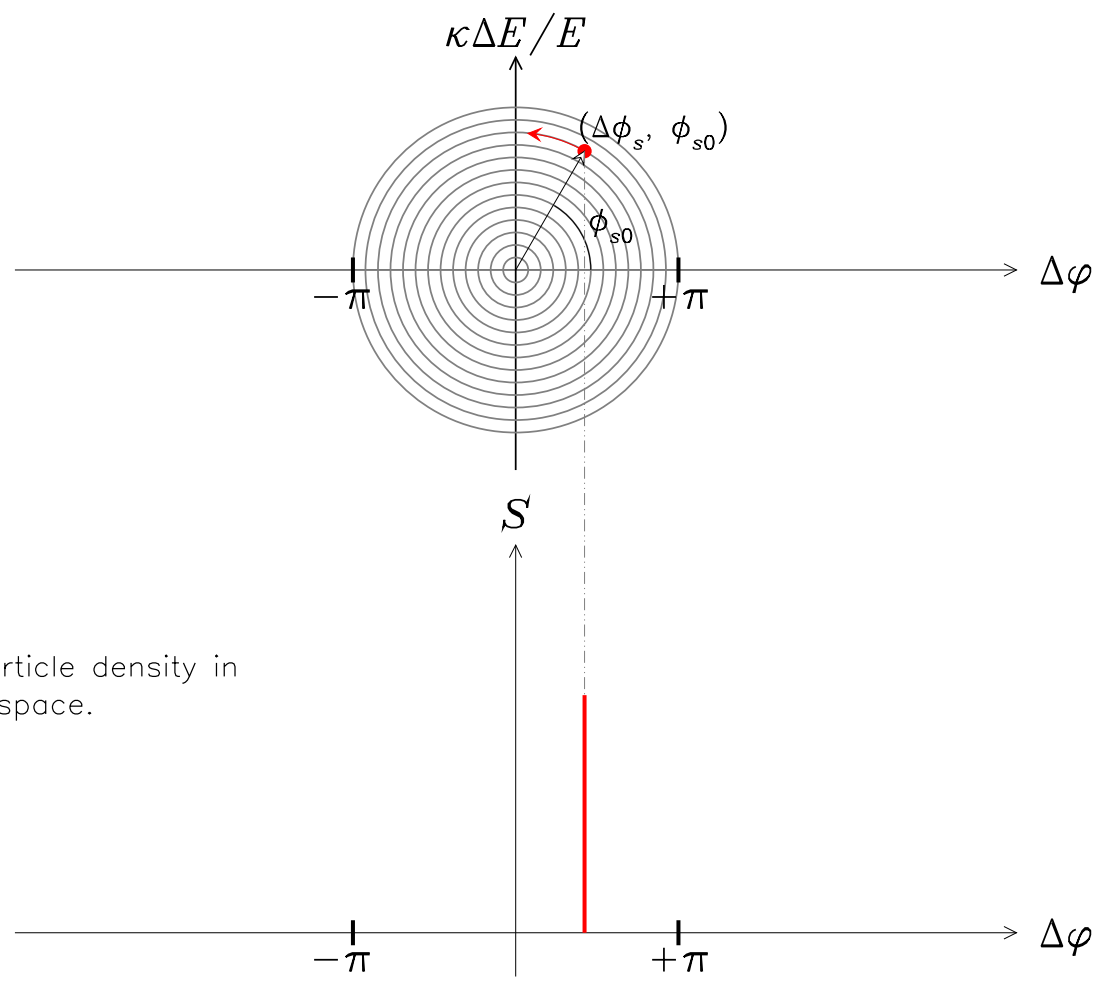

$S$ is the particle density in longitudinal space.

Figure 15 This figure shows the RF bucket where the $\delta$-function bunch resides. We have assumed that the bucket has a quadratic potential which means that the synchrotron frequency is constant. For example, at $t=0$, the bunch (coloured red) is at $\left(\Delta \phi_{s}, \phi_{s 0}\right)$ and when projected onto the $\Delta \varphi$ axis, the bunch is at $\Delta \phi_{s} \cos \phi_{s 0}$. At any other time, the bunch is at $\left(\Delta \phi_{s}, \Omega_{s} t+\phi_{s 0}\right)$ in phase space.

non-zero $\Delta p / p$, then its betatron tune has to include chromatic effects i.e.

$$
\begin{aligned}
Q & =Q_{0}+\xi \frac{\Delta p}{p} \\
& =Q_{0}+\frac{\xi \Omega_{s}}{\eta \omega_{\mathrm{rev}}} \Delta \phi_{s} \sin \left(\Omega_{s} t+\phi_{s 0}\right)
\end{aligned}
$$

where $Q_{0}$ is the betatron tune of the particle with zero chromaticity.

By definition, the betatron tune $Q$ is

$$
Q=\frac{\dot{\phi}_{Q}}{\dot{\varphi}_{k}}
$$


where $\dot{\phi}_{Q}$ is the betatron frequency. Substituting (58) and (60) into (61), we have

$$
\left.\begin{array}{rl}
\dot{\phi}_{Q} & =\left[Q_{0}+\frac{\xi \Omega_{s}}{\eta \omega_{\mathrm{rev}}} \Delta \phi_{s} \sin \left(\Omega_{s} t+\phi_{s 0}\right)\right] \times\left[\omega_{\mathrm{rev}}-\Omega_{s} \Delta \phi_{s} \sin \left(\Omega_{s} t+\phi_{s 0}\right)\right] \\
& =\omega_{\mathrm{rev}} Q_{0}-\Omega_{s} \Delta \phi_{s}\left(Q_{0}-\frac{\xi}{\eta}\right) \sin \left(\Omega_{s} t+\phi_{s 0}\right)-\frac{\xi}{\eta \omega_{\mathrm{rev}}} \Omega_{s}^{2} \Delta \phi_{s}^{2} \sin ^{2}\left(\Omega_{s} t+\phi_{s 0}\right)
\end{array}\right\}
$$

Integrating (62) once, we get the betatron phase

$$
\left.\begin{array}{rl}
\phi_{Q} & =\omega_{\mathrm{rev}} Q_{0} t+\Delta \phi_{s}\left(Q_{0}-\frac{\xi}{\eta}\right) \cos \left(\Omega_{s} t+\phi_{s 0}\right)+\phi_{k 0} \\
& =\theta_{Q} \tau+\Delta \phi_{s}\left(Q_{0}-\frac{\xi}{\eta}\right) \cos \left(\theta_{s} \tau+\phi_{s 0}\right)+\phi_{k 0}
\end{array}\right\}
$$

where $\tau=t / T_{\text {rev }}, T_{\text {rev }}$ is the revolution frequency of the synchronous particle, $\theta_{Q}=2 \pi Q_{0}$, $\theta_{s}=2 \pi \Omega_{s} / \omega_{\text {rev }}$, and $\phi_{k 0}$ is the betatron phase at $t=0$ and we have assumed that $\xi \Omega_{s}^{2} \Delta \phi_{s}^{2} / \eta \omega_{\text {rev }} \ll 1$. Thus from here, we can see how we can include the synchrotron term into the single particle quadrupole moment.

The single particle quadrupole moment $d q_{2}$ which we have found for coasting beam from (39), is easily modified to include the synchrotron term by using equation (63) and $\delta$-function terms which take into account the periodicity of the $\delta$-function bunch at the quadrupole pickup. The result is

$$
\begin{aligned}
d q_{2}=\zeta & \frac{a_{k 0}^{2}}{b^{2}}\left(\frac{\beta_{p}}{\beta_{k}}\right) \cos 2\left[\left(\theta_{Q}-\delta \theta_{k}\right) \tau+\Delta \phi_{s}\left(Q_{0}-\frac{\xi}{\eta}\right) \cos \left(\theta_{s} \tau+\phi_{s 0}\right)+\phi_{k p}\right] \times \\
& \sum_{n=-\infty}^{\infty} \delta\left(\varphi_{p}(t)-2 n \pi-\varphi_{p 0}\right)
\end{aligned}
$$

where $\zeta$ is a constant of proportionality which we will determine later, $\varphi_{p}$ is the azimuthal position of the bunch at the pickup (c.f. (57)) and $\varphi_{p 0}$ is the azimuthal position of the particle at $t=0$. We have dropped $\phi_{k 0}$ because it can be absorbed into $\phi_{k p}$ and the $\tan ^{-1}[$.$] term because we will be making the same approximation that we have used to de-$ rive (42). Note: $d q_{2}$ is dimensionless, but the dimensions of $\delta$-functions here are $1 /$ radians, and thus it is necessary to introduce $\zeta$ which has dimensions of radians to compensate. 
We note that the infinite sum of $\delta$-functions is also an infinite sum of cosines

$$
\sum_{n=-\infty}^{\infty} \delta\left(\varphi_{p}(t)-2 n \pi-\varphi_{p 0}\right)=\frac{1}{2 \pi} \sum_{n=-\infty}^{\infty} \cos n\left(\varphi_{p}(t)-\varphi_{p 0}\right)
$$

Note: we do not sum the cosines on the rhs because we want to preserve the negative frequency components in Fourier space so that we can directly read off modes later on. And so when this is substituted into (64), we get $\cos ($.$) terms multiplying \cos ($.$) terms$ which can be collapsed into sum of $\cos ($.$) terms by using the usual trigonometric formulæ.$ Thus $d q_{2}$ becomes

$$
d q_{2}=\zeta \frac{a_{k 0}^{2}}{4 \pi b^{2}}\left(\frac{\beta_{p}}{\beta_{k}}\right) \sum_{n=-\infty}^{\infty} \cos \theta_{n+}+\cos \theta_{n-}
$$

where

$$
\theta_{n \pm}=\left(2 n \pi \pm 2\left(\theta_{Q}-\delta \theta_{k}\right)\right) \tau+\left[n \pm 2\left(Q_{0}-\frac{\xi}{\eta}\right)\right] \Delta \phi_{s} \cos \left(\theta_{s} \tau+\phi_{s 0}\right)-n \varphi_{p 0} \pm 2 \phi_{k p}
$$

It is also well-known that terms of the form $\cos (Z \sin \theta)$ and $\sin (Z \sin \theta)$ can be expanded in terms of Bessel functions $J_{n}(z)$

$$
\left.\begin{array}{l}
\cos (Z \sin \theta)=\sum_{m=-\infty}^{\infty} J_{m}(Z) \cos m \theta \\
\sin (Z \sin \theta)=\sum_{m=-\infty}^{\infty} J_{m}(Z) \sin m \theta
\end{array}\right\} \quad \text { if } Z \in \mathbb{R}
$$

Therefore, (66) expanded in terms of Bessel functions is

$$
\left.\begin{array}{rl}
d q_{2}=\zeta \frac{a_{k 0}^{2}}{4 \pi b^{2}}\left(\frac{\beta_{p}}{\beta_{k}}\right) \sum_{n=-\infty}^{\infty}\left(\sum_{m=-\infty}^{\infty} J_{m}\left(Z_{n+}\right) \cos \left(\Theta_{n m+}+\psi_{n+}\right)+\right. & \left.\sum_{m=-\infty}^{\infty} J_{m}\left(Z_{n-}\right) \cos \left(\Theta_{n m-} \tau+\psi_{n-}\right)\right)
\end{array}\right\}
$$

where

$$
\left.\begin{array}{rl}
Z_{n \pm} & =\left[n \pm 2\left(Q_{0}-\frac{\xi}{\eta}\right)\right] \Delta \phi_{s} \\
\Theta_{n m \pm} & =2 n \pi \pm 2\left(\theta_{Q}-\delta \theta_{k}\right)+m \theta_{s} \\
\psi_{n m \pm} & =-n \varphi_{p 0}+m\left(\frac{1}{2} \pi+\phi_{s 0}\right) \pm 2 \phi_{k p}
\end{array}\right\}
$$




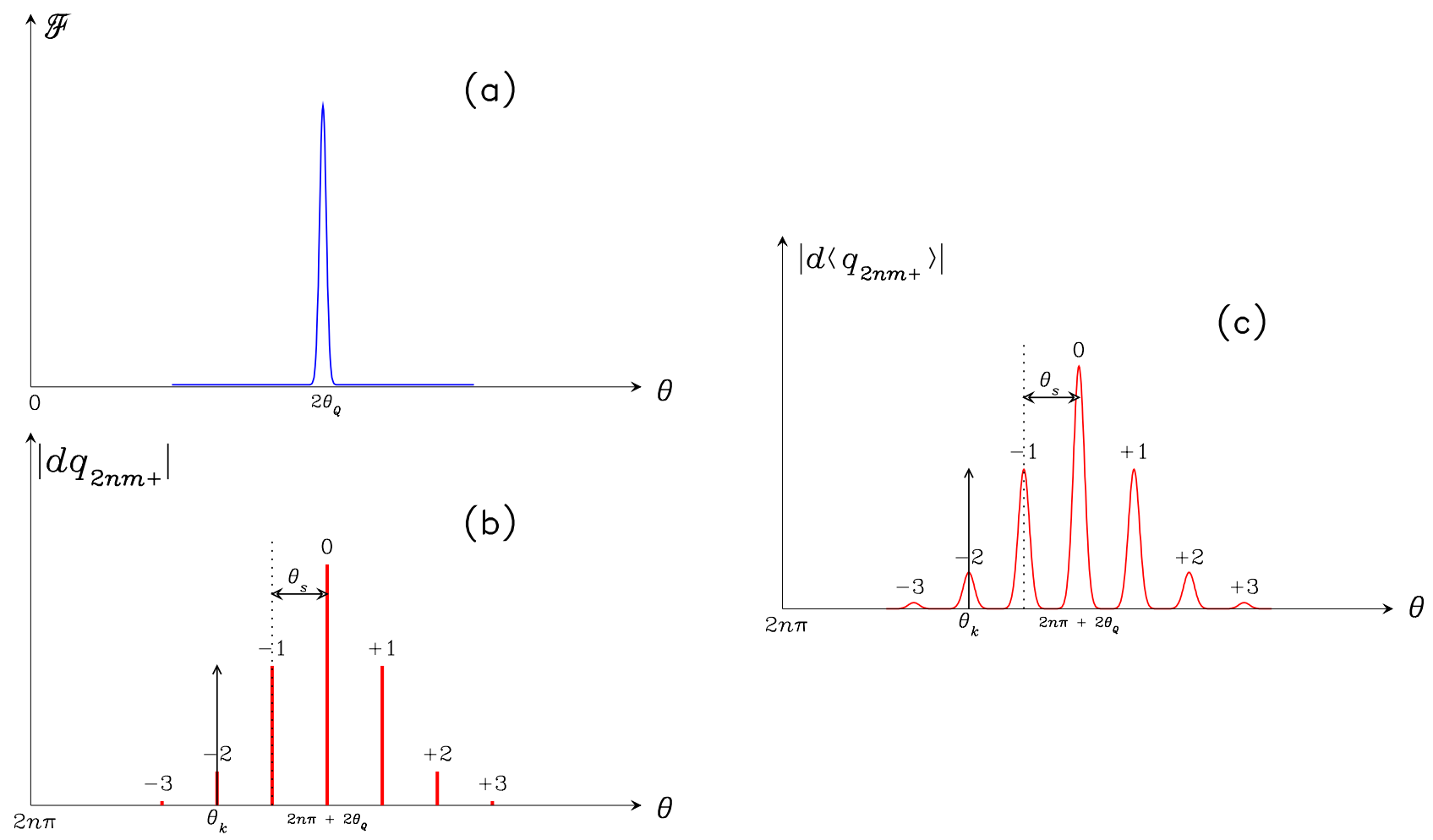

Figure 16 These series of spectra show how $\left|\mathcal{H}_{n m+}\right|$ is calculated. (a) shows a hypothetical $\mathcal{F}$ distribution at the quadrupole tune $2 \theta_{Q}$. (b) shows the spectrum of discrete lines around $2 n \pi+2 \theta_{Q}$ described by (69). Here, we show $\theta_{k}$ kicking at the resonance labelled by $(n,-2,+)$. (c) shows $\left|\left\langle d q_{2, n m+}\right\rangle\right|$ which is the result of "summing" over single particle combs shown in (b) weighted by $\mathcal{F}$. This means that the width of each synchrotron line is the width of the quadrupole tune distribution.

The interpretation of these equations is that there are an infinite number of resonances because of synchrotron motion which is unlike the coasting beam case which has only one resonant frequency. See Figure 16(b).

Next, we want to determine the value of $\zeta$. Let us choose one of the synchro-quadrupole resonances labelled by the quantum numbers $(n, m,+)$ to analyse. The arguments which we present here work equally well for $(n, m-)$. With this choice, we have

$$
d q_{2, n m+}=\zeta \frac{a_{k 0}^{2}}{4 \pi b^{2}}\left(\frac{\beta_{p}}{\beta_{k}}\right) J_{m}\left(Z_{n+}\right) \cos \left(\Theta_{n m+} \tau+\psi_{n m+}\right)
$$


Let us consider the special case when $\Delta \phi_{s}=0$, i.e. the bunch is matched to the bucket. With this condition, $Z_{n+}=0$ and thus $J_{0}(0)=1$ and $J_{m}(0)=0$ for $m \neq 0$ and so there are only resonances at $m=0$. For this special case, we can apply the results shown in Appendix II and equate the magnitude

$$
\left|d q_{2, n 0+}\right|=\zeta \frac{a_{k 0}^{2}}{4 \pi b^{2}}\left(\frac{\beta_{p}}{\beta_{k}}\right)
$$

to the magnitude

$$
\left|d q_{2}\right|=\frac{a_{k 0}^{2}}{b^{2}}\left(\frac{\beta_{p}}{\beta_{k}}\right)
$$

from (41) for coasting beam. Hence, $\zeta=4 \pi$.

\section{Including $\mathcal{F}$ Distribution}

Let us continue with the same synchro-quadrupole resonance $(n, m,+)$ to analyse but with $\Delta \phi_{s}$ no longer zero. Substituting $\zeta=4 \pi$ into (71), we have

$$
d q_{2, n m+}=\frac{a_{k 0}^{2}}{b^{2}}\left(\frac{\beta_{p}}{\beta_{k}}\right) J_{m}\left(Z_{n+}\right) \cos \left(\Theta_{n m+} \tau+\psi_{n m+}\right)
$$

For the QFR, we will kick at resonance i.e. when $\Theta_{n m+}=2 n \pi+2 \theta_{Q}+m \theta_{s} \equiv \theta_{k}$. See Figure 16(b) and thus (74) becomes

$$
d q_{2, n m+}=\frac{a_{k 0}^{2}}{b^{2}}\left(\frac{\beta_{p}}{\beta_{k}}\right) J_{m}\left(Z_{n+}\right) \cos \left(\theta_{k} \tau+\psi_{n m+}\right)
$$

For many particles, we have a distribution of $\theta_{Q}$ 's which is described by $\mathcal{F}(a, \theta)$. Like in the coasting beam calculation, if we assume that the particles which contribute to the quadrupole moment are in the region $\pm \epsilon / 2$ around $\theta_{k}$ (See Figure 11), then

$$
d\left\langle q_{2, n m+}\right\rangle=\epsilon \mathcal{F}\left(a_{k 0}, \theta_{k}-2 n \pi-m \theta_{s}\right) \times \frac{a_{k 0}^{2}}{b^{2}}\left(\frac{\beta_{p}}{\beta_{k}}\right) J_{m}\left(Z_{n+}\right) \cos \left(\theta_{k} \tau+\psi_{n m+}\right)
$$

Therefore, the contribution from all the particles is

$$
\left\langle q_{2, n m+}\right\rangle=\epsilon \frac{2 \pi}{b^{2}}\left(\frac{\beta_{p}}{\beta_{k}}\right) J_{m}\left(Z_{n+}\right) \int_{0}^{\infty} d a a^{3} \mathcal{F}\left(a, \theta_{k}-2 n \pi-m \theta_{s}\right) \mathcal{A}(a) \times \cos \left(\theta_{k} \tau+\psi_{n m+}\right)
$$


where $\mathcal{A} a d a d \phi$ is the stationary phase space density in $(a, \phi)$ space as before.

From here, like in the coasting beam case, we can easily read off the quadrupole magnitude response $\left|\mathcal{H}_{n m+}\right|$ for mode $(n, m,+)$ from $(77)$ and it is

$$
\left|\mathcal{H}_{n m+}\right|=\frac{2 \pi}{b^{2}}\left(\frac{\beta_{p}}{\beta_{k}}\right)\left|J_{m}\left(Z_{n+}\right)\right| \int_{0}^{\infty} d a a^{3} \mathcal{F}\left(a, \theta_{k}-2 n \pi-m \theta_{s}\right) \mathcal{A}(a)
$$

\section{Emittance}

In the prescription that we will use to extract the emittance from the QFR, we have to put one constraint on the $|\mathcal{H}|$ spectrum: we want the resonances to not overlap, i.e. the tune spread is smaller than the distance $\theta_{s}$ between the resonances. With this constraint, we can employ a similar trick like before. First, we select the synchro-quadrupole resonances with the same quantum numbers $n$ and + . Second, we integrate over $\theta_{k}$ for each synchrotron resonance $m$ to get rid of the $\mathcal{F}$ dependence and third, we sum the square of the contribution from each synchrotron resonance $m$ to get a result that only depends on the phase space distribution $\mathcal{A}$ and thus the emittance. See Figure 17.

Following the above prescription, we integrate out $\mathcal{F}$ for each synchrotron resonance. We can limit the integral to some small interval $\Delta$ centred around $\Theta_{n m+}$ because the synchrotron resonances do not overlap (See Figure 17(b)), i.e.

$$
\int_{0}^{\infty} d \theta \mathcal{F}\left(a, \theta-2 n \pi-m \theta_{s}\right)=\int_{\Theta_{n m+}-\Delta / 2}^{\Theta_{n m+}+\Delta / 2} d \theta \mathcal{F}\left(a, \theta-2 n \pi-m \theta_{s}\right)=1
$$

and therefore after integrating (78) over the small interval, we have a sequence of numbers

$$
d Q_{2, n m+}=\frac{2 \pi}{b^{2}}\left(\frac{\beta_{p}}{\beta_{k}}\right)\left|J_{m}\left(Z_{n+}\right)\right| \int_{0}^{\infty} d a a^{3} \mathcal{A}(a)
$$

Taking the square of of each number replaces $\left|J_{m}\right|$ with $J_{m}^{2}$ to give (See Figure $17(\mathrm{c})$ )

$$
\left(d Q_{2, n m+}\right)^{2}=\left[\frac{2 \pi}{b^{2}}\left(\frac{\beta_{p}}{\beta_{k}}\right) \int_{0}^{\infty} d a a^{3} \mathcal{A}(a)\right]^{2} J_{m}^{2}\left(Z_{n+}\right)
$$




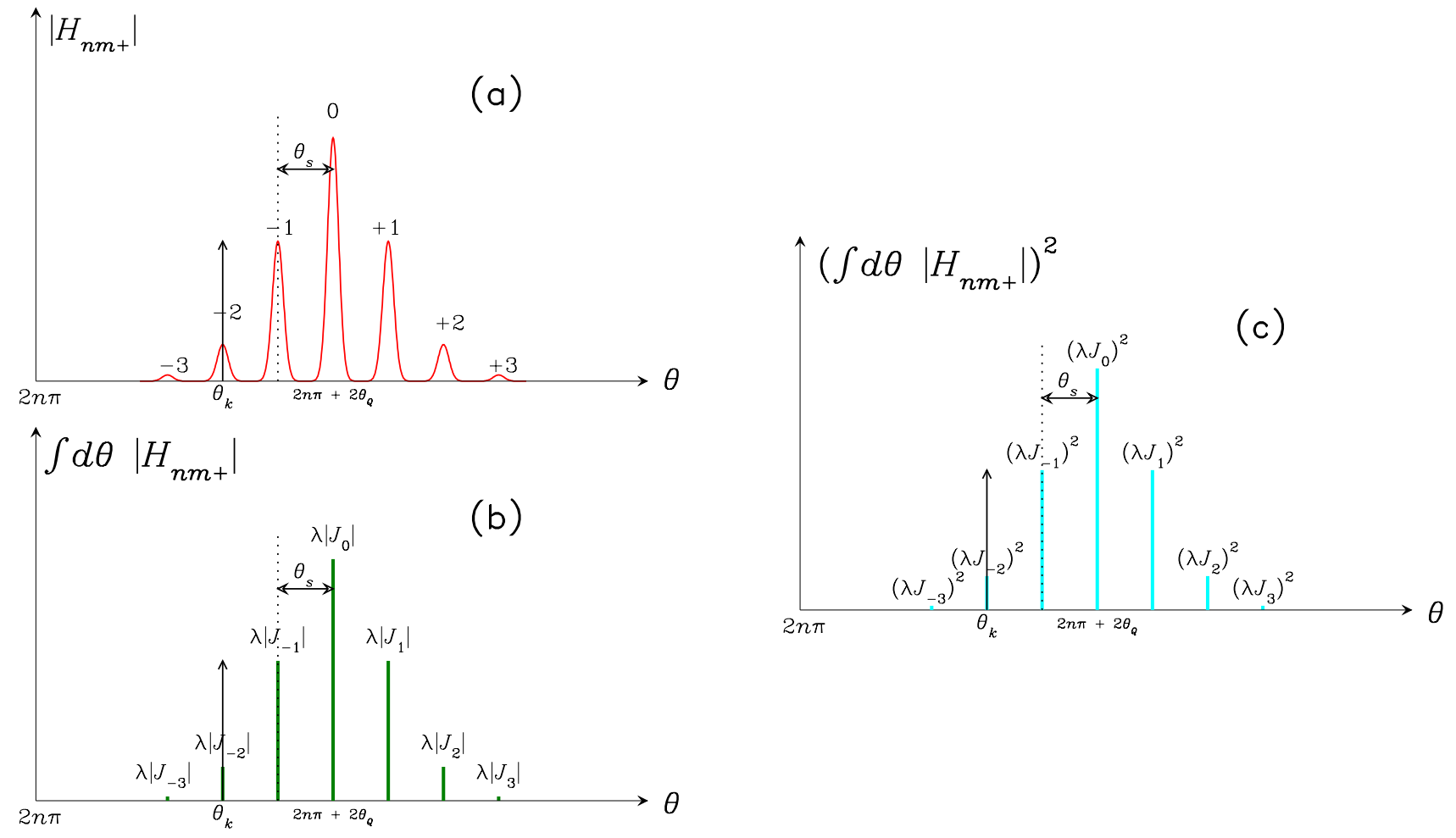

Figure 17 In this figure $\lambda=\frac{2 \pi}{b^{2}}\left(\frac{\beta_{p}}{\beta_{k}}\right) \int_{0}^{\infty} d a a^{3} \mathcal{A}(a)$. In (a) we have the synchrotron resonances well separated in the QFR. (b) Because the resonances are separated each resonance collapses to distinct numbers $\lambda\left|J_{m}\right|$ after $\mathcal{F}$ has been integrated out. (c) shows how by squaring each number we can replace $\left|J_{m}\right|$ with $J_{m}^{2}$.

Summing these numbers over $m$, we have

$$
\left(Q_{2, n+}\right)^{2}=\left[\frac{2 \pi}{b^{2}}\left(\frac{\beta_{p}}{\beta_{k}}\right) \int_{0}^{\infty} d a a^{3} \mathcal{A}(a)\right]^{2} \sum_{m=-\infty}^{\infty} J_{m}^{2}\left(Z_{n+}\right)
$$

We note that the sum ${ }^{11}$

$$
\sum_{m=-\infty}^{\infty} J_{m}^{2}(z)=1
$$

and thus (82) becomes independent of $Z_{n+}$ and consequently $\xi$

$$
\left(Q_{2, n+}\right)^{2}=\left[\frac{2 \pi}{b^{2}}\left(\frac{\beta_{p}}{\beta_{k}}\right) \int_{0}^{\infty} d a a^{3} \mathcal{A}(a)\right]^{2}
$$

Taking the square root of the above, we get

$$
Q_{2, n+}=\frac{2 \pi}{b^{2}}\left(\frac{\beta_{p}}{\beta_{k}}\right) \int_{0}^{\infty} d a a^{3} \mathcal{A}(a)
$$


For a bi-Gaussian distribution $\mathcal{A}$ with standard deviation $\sigma_{k}$, we have

$$
\left.\begin{array}{c}
Q_{2, n+}=8\left(\frac{\sigma_{k}}{b}\right)^{2}\left(\frac{\beta_{p}}{\beta_{k}}\right) \\
\Rightarrow \quad \sigma_{k}^{2}=\frac{1}{8} b^{2}\left(\frac{\beta_{k}}{\beta_{p}}\right) Q_{2, n+}
\end{array}\right\}
$$

Therefore, the transverse emittance $\varepsilon$ for a bi-Gaussian beam with standard deviation $\sigma_{k}$ is

$$
\varepsilon=\frac{6 \pi \sigma_{k}^{2}}{\beta_{k}}=\frac{3 \pi b^{2}}{4 \beta_{p}} Q_{2, n+}
$$

\section{Numerical Check IV}

In this section, the results of the previous section are numerically verified with the parameters shown in Table 5. We have drastically reduced $\sigma_{Q}$ from Numerical Check IIIa in order to ensure that the synchrotron resonances do not overlap. See Figure 18(a). The total number of turns used in the simulation has also been increased by a factor of 2 so that we can adequately resolve the synchrotron resonances because

$$
1 / N=1 / 16384=6.1 \times 10^{-5} \approx 29 \times\left(\frac{\theta_{s}}{2 \pi}\right)
$$

which means that there are 29 synchrotron periods in the time domain sample.

Figure 18(a) shows the process where we fit the synchrotron lines to Lorentzians. We apply the same fitting technique discussed in Check IIIa and ignore the DC term when we perform the integral. The other difference here is that we only integrate around each synchrotron resonance and not $\pm \infty$. Figure 18(b) shows the value of the integrals for each resonance. We follow the algorithm for calculating $\sigma_{k}$ from (81) to (85), where we square each integral value and sum, then take the square root and finally substitute the result into (86) to obtain $\sigma_{k}$ (calculated). 
Figure 19 shows the plot of $\sigma_{k}$ (calculated) versus $\sigma_{k}$ (input) for the parameters shown in Table 9 and the keys for deciphering the plot are shown in Table 6 . For the $\sigma_{Q}=$ $2 \pi \times 0.1 \times 10^{-4}$ distribution and $\xi=2$ or 5 , clearly $\sigma_{k}$ (input) $\approx \sigma_{k}($ calculated $)$, in fact the fits are

$$
\left.\begin{array}{l}
\sigma_{k}(\text { calculated })=(1.05 \pm 0.01) \sigma_{k}(\text { input }, \xi=2) \\
\sigma_{k}(\text { calculated })=(0.985 \pm 0.003) \sigma_{k}(\text { input }, \xi=5)
\end{array}\right\}
$$

It is a little poorer for $\xi=10$

$$
\sigma_{k}(\text { calculated })=(0.973 \pm 0.004) \sigma_{k}(\text { input }, \xi=2)
$$

Combining all the data for $\xi=2,5$ and 10, we get

$$
\sigma_{k}(\text { calculated })=(1.00 \pm 0.01) \sigma_{k}(\text { input }, \xi=2,5,10)
$$

which is exactly what we expect.

For $\sigma_{Q}=2 \pi \times 0.4 \times 10^{-4}$ and $\xi=5$ we find that $\sigma_{k}$ (calculate) is approximately $20 \%$ smaller than $\sigma_{k}($ input $)$

$$
\sigma_{k}(\text { calculated })=(0.80 \pm 0.01) \sigma_{k}(\text { input }, \xi=5)
$$

Part of the reason why the proportionality factor has deviated from 1 comes from the overlap of the synchrotron sidebands. See Figure 20.

\begin{tabular}{c|c|c|c}
\hline \multicolumn{2}{c}{ Table 5. Parameters used in Numerical Check IV for Mode $(\boldsymbol{4} \boldsymbol{m}+)$} \\
\hline $\begin{array}{c}\text { Parameter } \\
\theta_{Q}\end{array}$ & Value & Parameter & Value \\
$\theta_{s}$ & $2 \pi \times 20.575$ & $\xi$ & $2,5,10$ \\
$\epsilon$ & $2.5 \times 10^{-4}$ & $N_{A}$ & 16384 \\
$b$ & $1.5^{\prime \prime}$ & $M$ & 5 \\
$\beta_{p}=\beta_{k}$ & $10 \mathrm{~m}$ & $\sigma_{Q}$ & $20 \times 10^{3}$ \\
$\Delta \phi_{s}$ & $2 \times 10^{-4}$ & $\phi_{s 0}$ & $0.1,0.4) \times 10^{-4}$ \\
\hline \hline
\end{tabular}



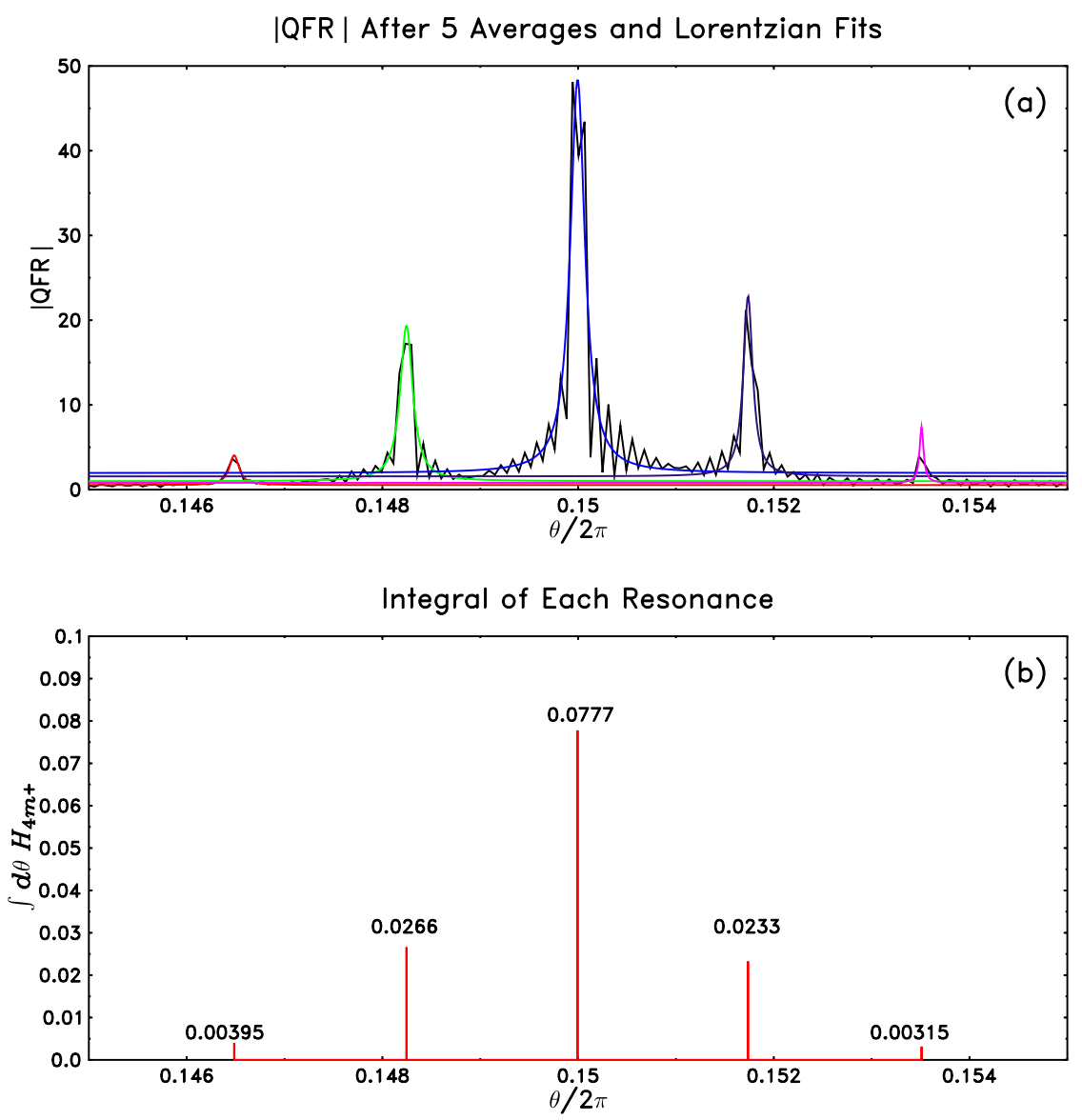

Figure 18 Here is the $(4, m,+)$ quadrupole mode for $\sigma=4 \mathrm{~mm}$, $\sigma_{Q}=2 \pi \times 0.1 \times 10^{-4}$ and $\xi=5$. (a) shows the Lorentzian fits for each resonance. (b) shows the results after integrating each Lorentzian. The value of each integral is written on top of each spike.

\begin{tabular}{c|c|c|c}
\hline \multicolumn{4}{c}{ Table 6. Keys for Deciphering the Markers in Figure 19 } \\
\hline Chromaticity $\boldsymbol{\xi}$ & Shape & $\boldsymbol{\sigma}_{\boldsymbol{Q}}$ & Colour \\
2 & $\triangle$ & $2 \pi \times 0.1 \times 10^{-4}$ & red \\
5 & $\bigcirc$ & $2 \pi \times 0.4 \times 10^{-4}$ & blue \\
10 & $\diamond$ & - & - \\
\hline \hline
\end{tabular}




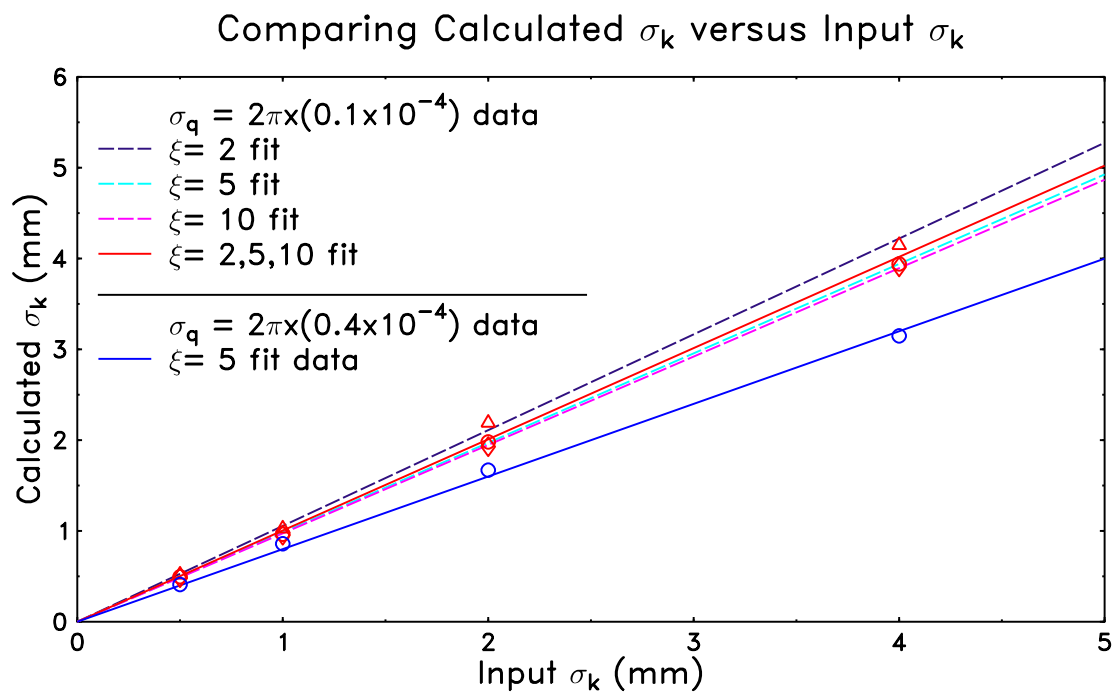

Figure 19 This graph compares the $\sigma_{k}$ used to create the normalised phase space and the calculated $\sigma_{k}$ from the QFR. The colours and shapes of the markers correspond to different chromaticities used in forming the initial distribution. See Table 6 for the keys needed for deciphering these markers. 

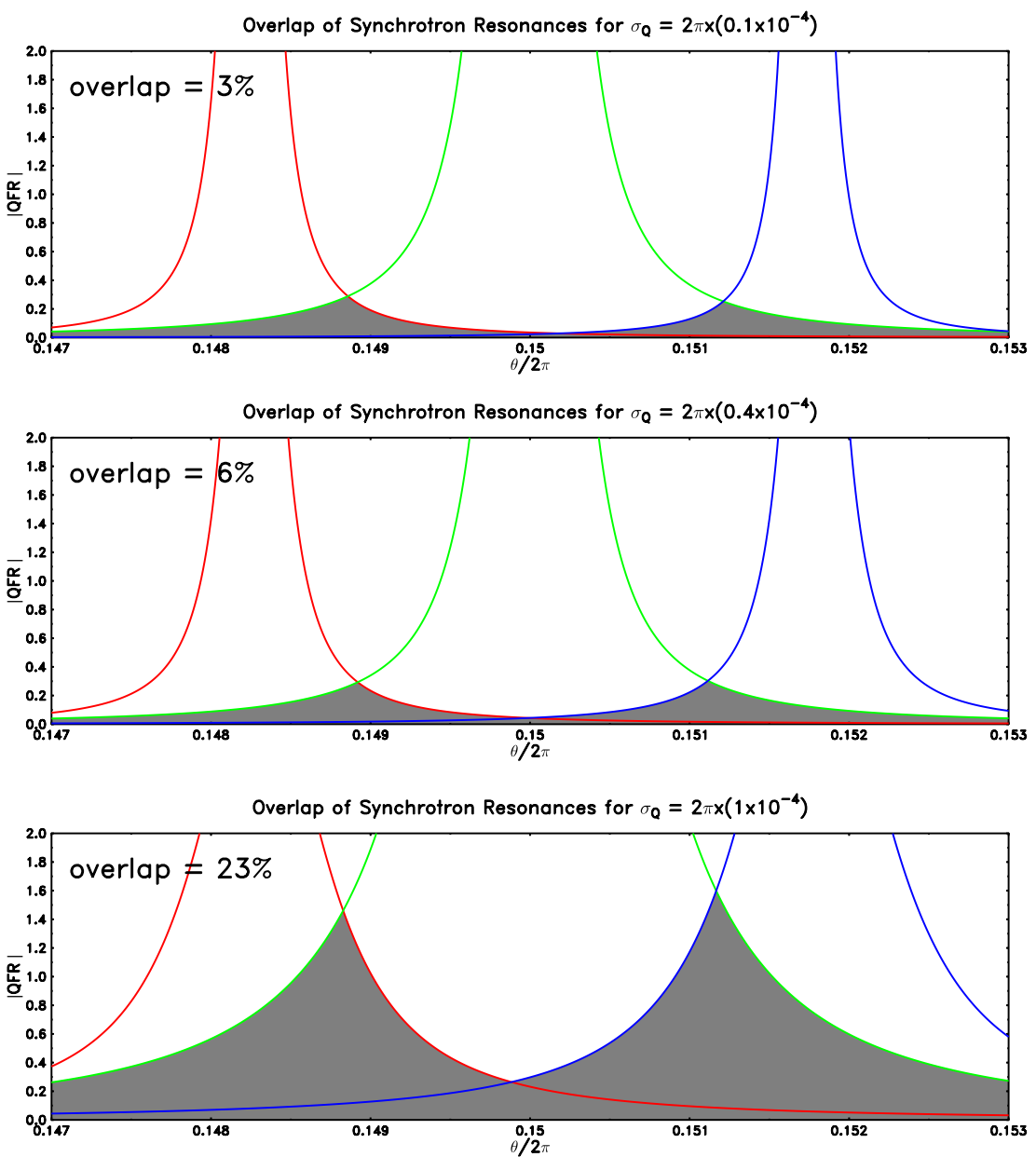

Figure 20 We compare the $|\mathrm{QFR}|$ obtained from $\sigma=4 \mathrm{~mm}$ and $\sigma_{Q}=2 \pi \times(0.1,0.4,1) \times 10^{-4}$. The synchrotron sidebands overlap more and more as $\sigma_{Q}$ increases. $\xi=5$ in all three cases. 


\section{CONCLUSION}

We have shown how the transverse emittance can be calculated from the QFR for very short bunches when the conditions that the quadrupole tunes are well decoupled and separated, and the quadrupole tune spread is smaller than the synchrotron tune have been met. Although we have written down an explicit emittance formula (78) and verified it for the bi-Gaussian in normalised transverse phase space case, we have not taken into account the frequency response of the kicker, pickup and electronics. Thus, in practice, unless all these frequency responses are known precisely, this method only gives relative emittances. Furthermore, we have also shown that the emittance will grow when the quadrupole kicker excites the beam continuously within the quadrupole tune distribution which, unfortunately, precludes a continuous measurement of the transverse emittance. However, we do not have actual experimental data to substantiate this claim or evidence as to whether this method can even work. Fortunately, this problem will be remedied soon because we have made plans to test this method out at RHIC where a quadrupole kicker and pickup are available for machine studies.

\section{ACKNOWLEDGEMENTS}

The author thanks P. Cameron for suggesting this method as a possible way to continuously measure emittance and A. Jansson for reading this paper. 


\section{APPENDIX I}

The focal length of a quadrupole kicker constructed using 4 striplines can be easily

calculated using Poisson. ${ }^{12}$ Figure 21 shows the cross section of the kicker which is assumed to be infinite in length. The parameters of the kicker used in the Poisson calculation are shown in Table 7. For this structure, the energy per unit length calculated by Poisson when the top and bottom plates are held at $+1 \mathrm{~V}$ and the left and right plates are held at $-1 \mathrm{~V}$ is $1.3154 \times 10^{-12} \mathrm{~J} / \mathrm{cm}$. The impedance $Z$ of each plate is given by

$$
Z=\left(2 c V^{2} \mathcal{E}\right)^{-1}
$$

where $c\left(=3 \times 10^{10} \mathrm{~cm} / \mathrm{s}\right)$ is the speed of light, $V(=1 \mathrm{~V})$ is the potential difference between the plate and the wall, and $\mathcal{E}\left(=1.3154 \times 10^{-12} / 4\right)$ is the energy stored by 1 plate and the wall. (Note: the "4" comes from dividing the energy among the four plates.) Putting the numbers into (93), we find that $Z=50.7 \Omega$. Therefore, we have a nice $50 \Omega \mathrm{RF}$ structure.

\begin{tabular}{l|c|c|c}
\hline Table 7. Parameters of the Quadrupole Kicker \\
\hline Parameter & Value & Parameter & Value \\
Outer Shell Diameter & $4^{\prime \prime}$ & Plate Radius & $1.5^{\prime \prime}$ \\
Angle of Plate & $52.5^{\circ}$ & Plate Thickness & $2 \mathrm{~mm}$ \\
\hline \hline
\end{tabular}

Next with the above potential differences, the E-field at $(0,1) \mathrm{cm}$ is $(0,-0.16) \equiv$ $\left(E_{x}, E_{y}\right) \mathrm{V} / \mathrm{cm}$, and thus for a stripline kicker of length $\ell=100 \mathrm{~cm}$, the positive angle of this kick per volt at the Tevatron injection energy $E_{n}=150 \mathrm{GeV}$ is

$$
\text { angle per volt }=\frac{2 E_{y} \ell}{E_{n}}=\frac{2 \times|0.16| \times 100}{150 \times 10^{9}}=2 \times 10^{-10} \mathrm{rad} / \mathrm{V}
$$

The focal length $f$ of this quadrupole kicker as a function of voltage is thus

$$
f(V)=\frac{1 \mathrm{~cm}}{\text { angle per volt } \times V}=\frac{1}{\left(2 \times 10^{-10}\right) V} \mathrm{~cm}
$$


If we assume that the kicker waveform is sinusoidal, and that the rms power $P$ going into the kicker is limited to $200 \mathrm{~mW}$, then the peak voltage $V_{p}$ on each plate is

$$
V_{p}=\sqrt{2(P / 4) Z}=\sqrt{2 \times 200 \times 10^{-3} / 4 \times 50} \approx 2 \mathrm{~V}
$$

where the "4" comes from dividing the power among the four plates. Therefore, the inverse focal length of the quadrupole kicker oscillates between $\pm\left(4 \times 10^{-10}\right) \mathrm{cm}^{-1}$ at $150 \mathrm{GeV}$.

Note: if we assume that the $\beta$-function at the kicker is approximately $\beta_{k}=10 \times 10^{2} \mathrm{~cm}$, then $\epsilon=\beta_{k} \delta F_{k}=\left(10 \times 10^{2}\right) \times\left(4 \times 10^{-10}\right)=4 \times 10^{-7}$. (For the examples in Numerical Check $I$ and $I I$ we have used $\epsilon$ which is three orders of magnitude bigger).

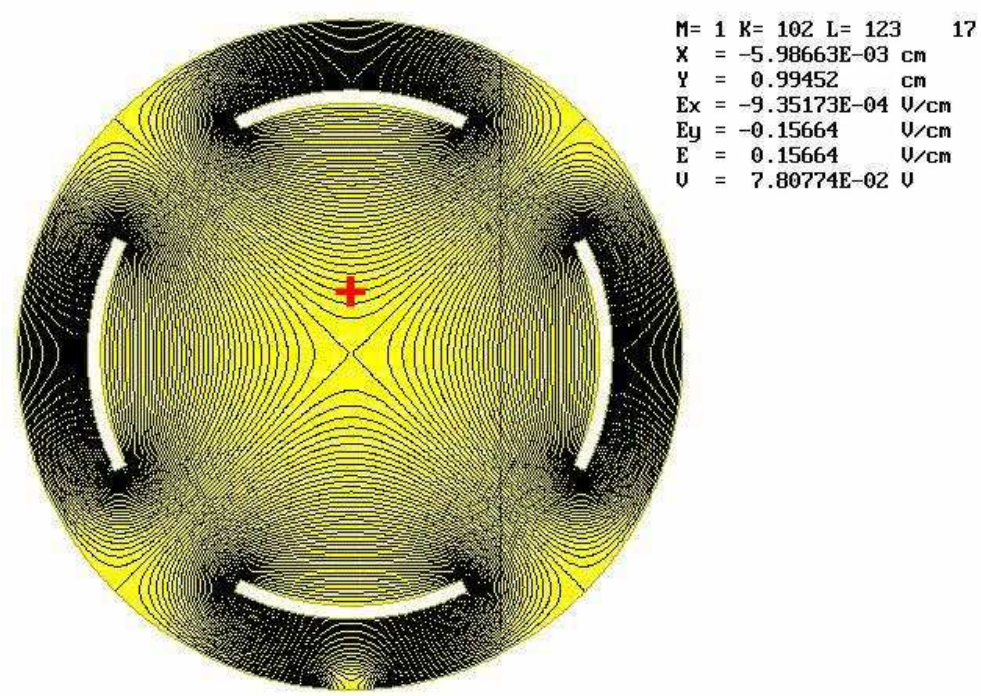

Figure 21 Here are the equipotential lines calculated by Poisson for a quadrupole kicker with transverse dimensions shown in Table 10. The point marked with a "+" is approximately at $(0,1) \mathrm{cm}$. The Efield at this point is $(0,-0.16) \mathrm{V} / \mathrm{cm}$. 


\section{APPENDIX II}

Suppose we have a continuous signal $f(\tau)$ and another signal described by

$$
g(\tau)=f(\tau) \sum_{n=-\infty}^{\infty} \delta(\tau-n)
$$

Let us define $F(\theta)$ and $G(\theta)$ to be the Fourier transforms of $f(\tau)$ and $g(\tau)$ respectively. We want to show that $G(\theta)$ in Fourier space consists of an infinite number of $F(\theta)$ 's spaced $2 \pi$ apart.

Let us define the Fourier transform to be

$$
F(\theta)=\int_{-\infty}^{\infty} d \tau f(\tau) e^{-i \theta \tau}
$$

Let the signal of interest be

$$
f(\tau)=\cos \theta_{Q} \tau
$$

The Fourier transform of $f(\tau)$ is ${ }^{13}$

$$
F(\theta)=\pi\left[\delta\left(\theta+\theta_{Q}\right)+\delta\left(\theta-\theta_{Q}\right)\right]
$$

The Fourier transform of $g(\tau)$ is

$$
\left.\begin{array}{rl}
G(\theta) & =\sum_{n=-\infty}^{\infty} e^{-i n \theta} \cos n \theta_{Q} \\
& =\frac{1}{2} \sum_{n=-\infty}^{\infty} e^{-i n\left(\theta+\theta_{Q}\right)}+e^{-i n\left(\theta-\theta_{Q}\right)}
\end{array}\right\}
$$

Using the Poisson sum formula

$$
\sum_{n=-\infty}^{\infty} e^{i n \theta}=2 \pi \sum_{n=-\infty}^{\infty} \delta(\theta-2 n \pi)
$$

and thus

$$
G(\theta)=\pi \sum_{n=-\infty}^{\infty} \delta\left(\theta+\theta_{Q}-2 n \pi\right)+\delta\left(\theta-\theta_{Q}-2 n \pi\right)=\sum_{n=-\infty}^{\infty} F(\theta-2 n \pi)
$$

This means that $G(\theta)$ consists of an infinite number of $F(\theta)$ spaced exactly $2 \pi$ apart. 


\section{REFERENCES}

[1] R.H. Miller et al, Nonintercepting Emittance Monitor, SLAC-PUB-3186, 1983.

[2] A. Jansson, Noninvasive Single-bunch Matching and Emittance Monitor, Phys. Rev. ST Accel. Beams 5, 072803 (2002).

[3] H. Koziol and K. Wittenburg, 4th ICFA Beam Dynamics Mini-Workshop on Transverse Emittance Preservation and Measurement, CERN preprint ps-98-002, 1997.

[4] Cameron et al, Quadrupole Transfer function for Emittance Measurement, TUPC08, DIPAC 2007.

[5] W. Guo and S.Y. Lee, Quadrupole-mode Transfer Function and the Nonlinear Mathieu Instability, Phys. Rev. E 65, 066505 (2002).

[6] M. Gasior and R. Jones, High Sensitivity Tune Measurement by Direct Diode Detection, CTWA01, DIPAC 2005.

[7] R.W. Brankin et al, RKSUITE Release 1.0, http: //www .netlib.org/ode/rksuite/ rksuite_90.zip, 1991.

[8] J.E. Clendenin et al, Proceedings of the 1981 Linear Accelerator Conference, 130, pg. 19-23, 1981.

[9] A.W. Chao, Physics of Collective Beam Instabilities in High Energy Accelerators, Chapter 5, John Wiley \& Sons Inc, 1993.

[10] D. McGinnis, Chromaticity Measurement Using Phase Modulated RF and Vector Signal Analyzers, Pbar Note 656, 2001.

[11] G.N. Watson, A Treatise on the Theory of Bessel Functions, pg. 31, Cambridge University Press, 2006.

[12] J.H. Billen and L.M. Young, Poisson Superfish, LA-UR-96-1834, 1998.

[13] S.J. Farlow, Partial Differential Equations for Scientists and Engineers, John Wiley \& Sons, 1982. 